\title{
T- $\beta$-MCA Regulates the Termination of Liver Regeneration via the P53/miR-34a/SIRT1 Positive Feedback Loop by Suppressing the FXR/SHP Signaling Pathway.
}

\section{Fangchao Yuan}

Chongqing Medical University https://orcid.org/0000-0003-1566-4400

\section{Yao Chen}

Chongqing Medical University

Hao Wu

Chongqing Medical University

Minghua Cong

Peking Union Medical College Hospital

\section{Menghao Wang}

Chongqing Medical University

\section{He Bai}

Xi'an Medical University

Jingyuan Wang

Chongqing

\section{Keting Que}

Dianjiang People's Hospital of Chongqing

Kaiwen Zheng

Dianjiang People's Hospital of Chongqing

\section{Wenfeng Zhang}

Chongqing Medical University

\section{Xiaoli Yang}

Southwest Medical University, Sichuan

Ke Xiao

Southwest Medical University, Sichuan

Hanping Shi

Capital Medical University

Mingyong Miao ( $\square$ miaomy@163.com )

Second Military Medical University

\section{Jianping Gong}

Chongqing Medical University 


\section{Research}

Keywords: liver regeneration, P53, miR-34a, SIRT1, HCC

Posted Date: November 16th, 2020

DOl: https://doi.org/10.21203/rs.3.rs-108116/v1

License: (c) (1) This work is licensed under a Creative Commons Attribution 4.0 International License. Read Full License 


\section{Abstract}

Background The capacity of the liver to restore its architecture and function assures good prognoses of patients who suffer serious hepatic injury or cancer resection. In our previous study, we initially found that the P53/miR-34a/SIRT1 positive feedback loop has a remarkable negative regulatory effect, which is related to the termination of liver regeneration. Here, we described how P53/miR-34a/SIRT1 positive feedback loop controls liver regeneration and its possible relationship with liver cancer.

Method We performed partial hepatectomy $(\mathrm{PH})$ in mice transfected with adenovirus (Ade) overexpressing P53 and adenovirus-associated virus (AAV) knock-downing miR-34a. LR was analyzed by liver weight/body weight, serum alanine aminotransferase (ALT) and aspartate aminotransferase (AST) levels and cell proliferation, and the related cellular signals were investigated. Bile acid (BA) levels during LR were analyzed by metabolomics of bile acids.

Results We found that the P53/miR-34a/SIRT1 positive feedback loop was activated in the late phase of LR. Overexpression of P53 terminated LR early and enhanced P53/miR-34a/SIRT1 positive feedback loop expression and its proapoptotic effect. Mice from the Ade-P53 group showed smaller livers and higher levels of serum ALT and AST than control mice. While knock-down of miR-34a abolished P53/miR34a/SIRT1 positive feedback loop during LR. Mice from anti-miR-34a group showed larger livers and lower levels of PCNA-positive cells than control mice. T- $\beta$-MCA increased gradually during LR and peaked at 7 days after $\mathrm{PH}$. T- $\beta$-MCA inhibited cell proliferation and promoted cell apoptosis via facilitating the P53/miR-34a/SIRT1 positive feedback loop during LR by suppressing FXR/SHP.

Conclusion The P53/miR-34a/SIRT1 positive feedback loop plays an important role in the termination of LR. Our findings shed light on the molecular and metabolic mechanisms of LR termination and provide a potential therapeutic alternative for treating P53-wild-type HCC patients.

\section{Introduction}

The astonishing regenerative capacity of the liver has been studied for decades. The capacity of the liver to restore its architecture and function assures good prognoses of patients who suffer serious hepatic injury, cancer resection, or living donor liver transplantation [1]. Thus, a better understanding of the mechanism involved in LR helps us gain insights into the cause of chronic and acute liver diseases and hepatocarcinogenesis. The complete process of LR, which includes initiation, proliferation and termination stages, requires the participation of numerous growth factors and cytokines, such as HGF, EGF, TGF- $\beta$, and TNF- $\alpha[2]$.

Although researchers have studied LR for decades, most of their work has focused on the initiation of LR, and little is known about the termination phase. Only a few studies have shed light on the mechanisms involved in the termination stage of LR. TGF- $\beta$ secreted by hepatocytes and nonparenchymal cells has been reported to inhibit DNA synthesis in regenerating hepatocytes [3]. Other studies have shown that IL$1 \mathrm{a}$ is another inhibitor of liver regeneration and suppresses DNA synthesis in hepatocytes [4]. Jin J et al. 
found that the cooperation between C/EBP family proteins and chromatin remodeling proteins plays important roles in the termination of LR [5]. A recent study reported that PP2Aca terminates LR in mice via the AKT/GSK3ß/Cyclin D1 signaling pathway [6]. Ian Huck found that knockout of hepatocyte nuclear factor 4 alpha increased hepatocyte proliferation throughout LR and that HNF4a activation was crucial for the termination of liver regeneration in mice[7].

A previous study by Miao confirmed that miR-34a was elevated in the late phase of LR after PHx in rats and suppressed the proliferation of hepatocytes during LR[8]. Furthermore, miR-34a-induced cell death might be related to the cellular context and the miRNA targets, which modulate apoptotic cell death. miR34a has been reported to be the direct target gene of P53[9]. Sirtuin 1 (Sirt1), which is a direct target of miR-34a, regulates the apoptosis of cells. Elevation of P53 acetylation and transcription induced by miR$34 a$ via the suppression of Sirt1 eventually leads to apoptosis. Thus, the P53/miR-34a/SIRT1 signaling pathway functions as a positive feedback loop, in which P53 activates miR-34a, while miR-34a induces acetylation and transcription of P53 by repressing SIRT1 to modulate cell apoptosis[10]. Theoretically, if not controlled, the proapoptotic effect of the P53/miR-34a/SIRT1 positive feedback loop would be reinforced infinitely. Jiyoung Lee et al. proved that the activation of miR-34a by P53 can be inhibited by the FXR/SHP signaling pathway[11]. FXR is a known receptor of bile acids (BAs). Therefore, we intend to explore to what extent the P53/miR-34a/SIRT1 positive feedback loop is involved in the termination of LR and whether there is a putative BA functioning as a regulatory factor of the P53/miR-34a/SIRT1 pathway through the FXR/SHP signaling pathway. Moreover, unlike the uncontrollable proliferation of liver cancer cells, normal liver regeneration can be terminated in time, and the size of the liver can be accurately controlled. Therefore, the disorder of the mechanism involved in the termination of liver regeneration may be related to the occurrence and development of liver cancer. We also explored the relationship between the $\mathrm{p} 53 / \mathrm{miR}-34 \mathrm{a} / \mathrm{SIRT} 1$ positive feedback loop and HCC.

\section{Materials And Methods}

Materials and Methods was shown in supplementary material.

\section{Results}

\section{The P53/miR-34a/SIRT1 proapoptotic pathway is activated in the termination stage of LR.}

To explore the function of P53/miR-34a/SIRT1 during LR in mice, we first determined whether PH could activate the P53/miR-34a/SIRT1 positive feedback loop. P53 remarkably increased at both day 2 and day 7 after $\mathrm{PH}$, while no significant change was detected in miR-34a at day 2 of PH (Fig. 1A, C). Thus, P53induced activation of the P53/miR-34a/SIRT1 positive feedback loop might be inhibited by certain mechanisms. The level of acetylated P53 was in accordance with miR-34a expression. SIRT1 decreased gradually from day 2 to day 7 after $\mathrm{PH}$ and increased thereafter (Fig. 1A, C). As LR progressed, miR-34a expression gradually increased, peaked at day 7 after $\mathrm{PH}$ and decreased sharply thereafter (Fig. 1B). 
Therefore, we postulated that the P53/miR-34a/SIRT1 positive feedback loop is activated in the late stage of $\mathrm{PH}$ and might stimulate LR termination.

\section{Overexpression of wild-type P53 terminates LR and activates the P53/miR-34a/SIRT1 positive feedback loop early during LR in vivo.}

To further examine the function of the P53/miR-34a/SIRT1 positive feedback loop in LR, we performed $\mathrm{PH}$ on mice that received tail-vein injection of adenovirus overexpressing P53 (Figure S1). The size of regenerated livers in the Ade-P53 group was significantly smaller than that of mice in the Ade-GFP group (Fig. 2A). The liver-to-body weight ratio at day 7, day 10 and day 14 was significantly lower in the Ade-P53 group, and the liver-to-body weight ratio stopped increasing starting on day 5 in the Ade-P53 group (Fig. 2B). Then, we compared the proliferation of cells in the liver during LR, and the PCNA-positive cells were higher in the Ade-GFP group on day 2 and 3 after PH (Fig. 2C, D). We investigated the serum levels of ALT and AST, which are considered two indicators of liver damage after PH. The serum levels of ALT and AST increased immediately after surgery and then decreased gradually as the liver mass and physiological structures recovered in Ade-GFP group. However, the serum levels of ALT and AST were significantly higher and did not recover to normal in Ade-P53 mice compared with GFP mice (Fig. 2E, F). The survival rate of mice that received PH surgery decreased sharply to 50\% in the Ade-P53 group (Fig. 2G). TUNELpositive cells in liver increased in P53 group (Fig. 2H, I). When P53 was overexpressed, the P53/miR34a/SIRT1 positive feedback loop was activated in the Sham, day 2 and day 7 groups (Fig. 2J-L). Therefore, these data suggest that knock-in of P53 activates the P53/miR-34a/SIRT1 positive feedback loop during LR and terminates LR early.J-L

\section{Knock-down of miR-34a de-activates the P53/miR-34a/SIRT1 positive feedback loop during LR and inhibits LR termination in vivo.}

To further examine the function of the P53/miR-34a/SIRT1 positive feedback loop in LR, we performed $\mathrm{PH}$ on mice that received tail-vein injection of AAV that down-regulated miR-34a (Figure S2). The size of regenerated livers in the anti-miR-34a group was significantly bigger than that of mice in the AAV-GFP group at day 7 after PH (Fig. 3A). The liver-to-body weight ratio at day 5, 7, 10 and 14 were significantly higher in the anti-miR-34a group (Fig. 3B). We next assessed cell proliferation by PCNA staining, and cell proliferation in the liver was promoted in the anti-miR-34a group at 3, 5, 7, 10 days after PH (Fig. 3C, D). TUNEL-positive cells in liver decreased sharply in anti-miR-34a group (Fig. 3E, F). When miR-34a was knocked down, the P53/miR-34a/SIRT1 positive feedback loop was down-regulated, as were the proapoptotic genes downstream (Fig. 3G, H). Therefore, these data suggest that knock-down of miR-34a abolished the P53/miR-34a/SIRT1 positive feedback loop during LR and LR could not be terminated then.

\section{T- $\beta$-MCA enhanced the proapoptotic effect of the P53-activated P53/miR-34a/SIRT1 positive feedback loop in primary mouse hepatocytes by suppressing the FXR/SHP signaling pathway.}

It has been proven that BAs participate in LR by acting as signaling molecules that activate signaling pathways $[12,13]$. Previous studies have confirmed a stimulatory role of BA at physiological 
concentrations in LR $[14,15]$. Here, we analyzed BAs in the regenerated liver tissue of mice. In our study, we found that there was a difference in the activation of the P53/miR-34a/SIRT1 positive feedback loop induced by the overexpression of P53 between the early and late stages of LR (Fig $2 \mathrm{I}, \mathrm{J}$ ). Overexpression of P53 increased the P53 level at 2 days after PH to a level higher than the P53 level at 7 days after PH in the Ade-GFP group, while the miR-34a level was lower. Therefore, P53 transactivation of miR-34a was inhibited. We postulated that this might be due to the change in the BA pool during LR.

We performed metabolomics of BAs during LR, which included 22 BAs in total, by UHPLC (Table S1). Among these BAs, 13 changed significantly during LR in our study (Fig 4A). The levels of 5 BAs were elevated during LR compared with the Sham group. The levels of 8 BAs decreased in the latter phase of LR. Among the top 3 BAs with the highest concentrations, T- $\beta-M C A$ increased gradually during LR (Fig $4 B$ ). We observed that the level of T- $\beta$-MCA was correlated with the level of miR-34a. As LR progressed, the level of T- $\beta$-MCA gradually increased, peaked at day 7 after $\mathrm{PH}$ and decreased sharply thereafter (Fig 4C). Therefore, these data suggest that the P53/miR-34a/SIRT1 positive feedback loop might be manipulated by T- $\beta$-MCA.

Beta-muricholic acid (bMCA) is a major BA in rats and mice [16]. In a recent major study, Fredrik Backhed and his colleagues proved that tauro- $\beta$-muricholic acid (T- $\beta-M C A)$ is a competitive and reversible FXR antagonist and that treatment with T- $\beta$-MCA reduces TCA-induced activation of the FXR/SHP signaling pathway[17]. Activating the FXR/SHP signaling pathway can inhibit the transcriptional activation of miR34a by P53[11]. Therefore, we hypothesized that T- $\beta$-MCA is able to enhance the effect of the P53activated P53/miR-34a/SIRT1 positive feedback loop by suppressing the FXR/SHP signaling pathway.

We transfected primary mouse hepatocytes with adenovirus carrying wild-type P53. The transfection efficiency was verified by WB and fluorescent imaging (Figure S3). In our study, TUNEL staining showed that the apoptosis of hepatocytes was enhanced by T- $\beta$-MCA (Fig 4D, E). We found that P53 overexpression activated the P53/miR-34a/SIRT1 positive feedback loop in primary mouse hepatocytes, while it was enhanced by the administration of T- $\beta$-MCA downstream of proapoptotic genes (Fig $4 \mathrm{G}-\mathrm{H})$. The FXR/SHP signaling pathway is inhibited by T- $\beta$-MCA treatment. Treatment with GW4064, an FXR/SHP signaling pathway agonist, was able to activate the FXR/SHP signaling pathway and inhibit the $\mathrm{P} 53 / \mathrm{miR}-34 \mathrm{a} / \mathrm{SIRT} 1$ positive feedback loop in primary mouse hepatocytes. When cells were co-incubated with T- $\beta-M C A$, the GW4064-induced activation of the FXR/SHP signaling pathway was inhibited.

\section{T- $\beta$-MCA facilitates the P53/miR-34a/SIRT1 positive feedback loop during LR by suppressing the FXR/SHP signaling pathway in mice.}

We next performed PH on mice fed a normal diet (ND) and mice fed extra T- $\beta$-MCA in sterile water by gavage. The size of regenerated livers in the T- $\beta$-MCA group was significantly smaller than that of mice in the ND group at 7 days after $\mathrm{PH}$ (Fig. 5A). The liver-to-body weight ratio at days 5, 7, 10 and 14 after $\mathrm{PH}$ was significantly lower in the T- $\beta$-MCA group (Fig. 5B). PCNA staining showed that cell proliferation was suppressed in the T- $\beta$-MCA group (Fig. 5C, D). The serum levels of ALT and AST increased immediately after surgery and then decreased gradually as the liver mass and physiological structures recovered in ND 
group. However, the serum levels of ALT and AST were significantly higher and did not recover to normal in T- $\beta$-MCA mice compared with ND mice (Fig. 2E, F). The survival rate of mice that received PH surgery decreased sharply to $50 \%$ in the Ade-P53 group (Fig. 2G). TUNEL-positive cells in liver increased in T- $\beta$ MCA group (Fig. 5H, I). WB showed that the effect of the P53/miR-34a/SIRT1 positive feedback loop increased, while FXR/SHP was inhibited in the T- $\beta$-MCA group (Fig. $5 \mathrm{G}-\mathrm{H})$. Therefore, these data suggest that T- $\beta$-MCA is able to facilitate the proapoptotic effect of the P53-activated P53/miR-34a/SIRT1 positive feedback loop by suppressing the FXR/SHP signaling pathway and terminate LR early in vivo.

\section{Knock-in of wild-type P53 reinforce the P53/miR-34a/SIRT1 positive feedback loop in in P53-wild-type HepG2 cell lines.}

Hepatocellular carcinoma (HCC) is a malignant tumor and the third leading cause of cancer-related mortality[18]. Both LR and HCC are characterized by high cell proliferation, but there are significant differences between the two. The unlimited proliferation of HCC cells lacks a termination mechanism, while during LR, cell proliferation can be terminated, and liver mass is precisely regulated. Due to TP53 mutations, the P53 response pathway is frequently deficient in HCC patients[19,20]. Thus, we hypothesized that P53/miR-34a/SIRT1 fails to function during HCC due to the loss or mutation of P53.

Accordingly, we evaluated the functions and expression of the P53/miR-34a/SIRT1 positive feedback loop in vitro. To elucidate the effects of the P53/miR-34a/SIRT1 positive feedback loop on cell apoptosis and proliferation in vitro, we investigated liver cancer cells with different endogenous P53 expression levels, HepG2 (P53-wt), Hep3B (P53-de) and Huh7 (P53-mut) and overexpressed wild-type P53 with a plasmid[21] (Figure S4 A). The transfection efficiency was verified by WB and fluorescent imaging (Figure S4 B C). Only two CAs function as FXR antagonists, T- $\beta-M C A$ in mice and ursodeoxycholic acid (UDCA) in human[22,23]. In our experiment, we test the other FXR antagonist UDCA's effect on P53/miR-34a/SIRT1 positive feedback loop in human liver cancer cell as well[24].

The P53/miR-34a/SIRT1 positive feedback loop was detected in P53-wild-type HepG2 cells (Fig 6A-C). Administration of UDCA alone increased P53/miR-34a/SIRT1 positive feedback loop expression and its down-stream targets (Fig 6A-C). Overexpression of wild-type P53 enhanced P53/miR-34a/SIRT1 positive feedback loop expression and its pro-apoptotic effect in HepG2 cells and could be facilitated even more by administration of UDCA (Fig 6A-C). Overexpression of wild-type P53 increased TUNEL-positive cells and inhibited proliferation of HepG2 cells, and UDCA could improve its effect (Fig 6D, E).

\section{Knock-in of wild-type P53 restored the P53/miR-34a/SIRT1 positive feedback}

\section{in P53-mutated Huh-7 cell line.}

The P53/miR-34a/SIRT1 positive feedback loop was absent in P53-mutated Huh7 cells, while overexpression of wild-type P53 in Hep3B cells restored the P53/miR-34a/SIRT1 positive feedback loop and could be further enhanced by administration of UDCA (Fig 7A-C).Additionally, the FXR/SHP signaling pathway was inhibited by administration of UDCA in Huh7 cells(Fig 7A-C). Overexpression of wild-type 
P53 increased apoptosis and inhibited proliferation of Huh7 cells, and UDCA could improve its effect (Fig 7D, E).

\section{Knock-in of wild-type P53 restored the P53/miR-34a/SIRT1 positive feedback}

\section{in P53-deficient Hep-3B cell lines.}

The P53/miR-34a/SIRT1 positive feedback loop does not exist in P53-deficient Hep3B cells.

Overexpression of wild-type P53 in Hep3B cells restored the P53/miR-34a/SIRT1 positive feedback loop, which could be further enhanced by administration of UDCA (Fig 8A-C). Additionally, the FXR/SHP signaling pathway was inhibited by administration of UDCA in Hep3B cells (Fig 8A-C). Overexpression of wild-type P53 increased apoptosis and inhibited proliferation of Hep3B cells, and UDCA could improve its effect (Fig 7D, E).

\section{Discussion}

The liver is extremely vulnerable to physical and chemical damage. LR protects the liver by helping the liver recover from such damage. However, the exact mechanisms underlying the termination stage of LR after $\mathrm{PH}$ remain unclear. In this study, we demonstrated that the P53/miR-34a/SIRT1 positive feedback loop was significantly activated in the late stage of LR. Furthermore, overexpression of P53 increased apoptosis of hepatocytes during LR and ended LR beforehand, while knock-down of miR-34a abolished P53/miR-34a/SIRT1 positive feedback loop during LR and suppressed LR termination (Fig 2, 3). Here, we propose a new mechanism that regulates the termination of LR to enrich the understanding of the underlying regulatory mechanism. As the P53/miR-34a/SIRT1 positive feedback loop has been reported to be involved in blocking cell proliferation and inducing cell apoptosis in numerous cell lines and diseases, our research will provide new insights into the study of P53/miR-34a/SIRT1 in both LR and tumorigenesis.

TP53 is a well-known tumor suppressor gene that can be activated by DNA damage induced by radiation, oxidative stress and chemotherapeutic drugs[25,26]. Mutations in P53 have caused many human cancers, including HCC[27]. Several studies verified that P53 regulated the expression of the miR-34 miRNA family, including miR-34a, miR-34b and miR-34c. The expression of miR-34a coordinates cell cycle progression, senescence and apoptosis[28-31]. SIRT1 is crucial for the protection of cells against stress and DNA damage. SIRT1 deacetylates the Lys382 residue of P53 to suppress transcriptional activation of P53 and decrease its downstream proteins in an NAD+-dependent manner[32,33]. Yamakuchi and his colleagues overexpressed miR-34a and found decreased SIRT1 expression, resulting in increased acetylated P53 levels and increased levels of P53 targets such as p21 and PUMA[10]. This is how the P53/miR-34a/SIRT1 positive feedback loop functions. It has been shown that the P53/miR34a/SIRT1 positive feedback loop is activated according to disease severity in human non-alcoholic fatty liver disease (NAFLD), and several antitumor drugs could activate the P53/miR-34a/SIRT1 positive feedback loop in cancer cell lines to induce the apoptosis of cancer cells[34,35]. Duarte M. S. Ferreira et al. demonstrated that P53/miR-34a/SIRT1 activated by JNK1/c-Jun contributed to cell death caused by 
deoxycholic acid in rats[36]. In our study, the P53/miR-34a/SIRT1 positive feedback loop was activated in the late stage of LR, and its proapoptotic and antiproliferative effects could be necessary to terminate LR. Serum ALT and AST levels in mice after PH did not recover to normal in mice overexpressing P53, which might be due to liver regeneration in mice overexpressing P53 being terminated earlier than under normal conditions. Overexpression of P53 intensified the effect of the P53/miR-34a/SIRT1 positive feedback loop in the termination stage of LR; however, it failed to enhance the loop at 2 days after PH to the extent it did at 7 days after PH (Fig 2I). Our findings suggest that the P53/miR-34a/SIRT1 positive feedback loop is activated during the termination stage of LR rather than the initiation or proliferation stage. Although there was an increase in P53, it failed to stimulate the P53/miR-34a/SIRT1 positive feedback loop in the early phase of LR (Fig 1A). Thus, there might be a certain mechanism that controls the activation of the P53/miR-34a/SIRT1 positive feedback loop. Meanwhile, knocked-down of miR-34a suppressed the P53/miR-34a/SIRT1 positive feedback loop and its effect (Fig. 3G, H). LR became infinitely and could not be terminated during the first 14 days after $\mathrm{PH}$ (Fig. 3B). These data suggest P53/miR-34a/SIRT 1 positive feedback loop is essential for LR termination.

BAs are specifically synthesized from cholesterol in the liver. Digesting fat-soluble molecules in food and facilitating the absorption of lipids are two fundamental functions of BAs in vivo. It has been shown that BA is able to promote liver regeneration by activating the FXR/SHP signaling pathway[13]. However, the authors did not analyze the specific BA composition. The proportion of BA that promoted LR and activated the FXR/SHP signaling pathway remained unclear. In our study, we found that total BAs increased in the early phase of $L R$ and then decreased in the termination stage of $L R$, with a gradual increase in the proportion of T- $\beta$-MCA over total BA (Fig 4B, C). Sama I. Sayin et al. identified T- $\beta$-MCA as an FXR antagonist that inhibited the FXR/SHP signaling pathway[17]. Our study showed that T- $\beta-M C A$ suppressed the FXR/SHP signaling pathway and amplified the proapoptotic effects of P53-induced activation of the P53/miR-34a/SIRT1 positive feedback loop both in vitro and in vivo.

TP53 mutations are commonly found in HCC patients. Previous research showed that TP53 had the highest prevalence of protein-altering mutations in $\mathrm{HCC}[37,38]$. Several studies have shown that tumor drugs can suppress the proliferation of liver cancer cells by activating the P53/miR-34a/SIRT1 positive feedback loop[39,35]. In our study, we show that the P53/miR-34a/SIRT1 positive feedback loop is deficient in the P53-deficient Hep3B and P53-mutated Huh7 cell lines. The P53 level is higher in the P53mutated Huh7 cell line than in the HepG2 cell line, while the P53/miR-34a/SIRT1 positive feedback loop is not activated. After knock-in of wild-type P53, the P53/miR-34a/SIRT1 positive feedback loop was restored and further enhanced by administration of UDCA through suppressing FXR/SHP in all cell lines. Administration of UDCA alone in the wild-type P53 HepG2 cell line promoted the P53/miR-34a/SIRT1 positive feedback loop. Thus, deficiency in the P53/miR-34a/SIRT1 positive feedback loop induced by the mutation or loss of P53 might be the cause of HCC tumorigenesis and development. Additionally, UDCA or other alternative FXR agonists could be a potential therapeutic target for HCC patients with wild-type P53 expression. 


\section{Conclusion}

In summary, a P53/miR-34a/SIRT1 positive feedback loop exists and is activated in the termination of LR. The effect of the P53/miR-34a/SIRT1 positive feedback loop is regulated by T- $\beta$-MCA/UDCA through the FXR/SHP signaling pathway, and the mechanism is summarized in the diagram (Graphical Abstract). These findings may provide new insight into the treatment of chronic liver diseases, liver transplantation, and liver malignancy, especially for HCC patients with wild-type P53 expression.

\section{Abbreviations}

ALT: alanine transaminase; AST: aspertate aminotransferase; AAV: adenovirus associated virus; Ade: adenovirus; BA: bile acid; FXR: farnesoid x receptor; GFP: green fluorescent protein; HCC: hepatocellular carcinoma; IHC: immunohistochemistry; LR: liver regeneration; MCA: muricholic acid; T- $\beta-M C A$ : tauro- $\beta$ muricholic acid; PH: partial hepatectomy; RFP: red fluorescent protein; SHP: small heterodimer partner; TUNEL: TdT-mediated dUTP nick end labeling; UDCA: ursodeoxycholic acid; UHPLC: ultrahighperformance liquid chromatography.

\section{Declarations}

\section{Ethics approval and consent to participate}

Ethical reviews of animal experiment were approved by the ethics committee of the Second Affiliated Hospital of Chongqing Medical university and all animals' care and experimental protocols were in line with the Animal Management Rules of the Ministry of Health of the People's Republic of China.

\section{Consent for publication}

The study was conducted in accordance with the protocol approved by the Declaration of Helsinki and the guidelines of the Ethics Review Committee of the Second Affiliated Hospital of Chongqing Medical University

\section{Availability of data and materials}

The data sets used and/or analyzed during the current study are available from the corresponding author on reasonable request.

\section{Competing interests}

All of the authors declare that there are no competing interests.

\section{Funding}

This study was supported by grants from the National Natural Science Foundation of China (NSFC: No.81670599; awarded to GJP; No. 81570569 awarded to MMY and No. 81672888 awarded to HPS) 
administered by the the National Natural Science Fund Committee. This study was supported by supported by the Kuanren Talents Program of the second affiliated hospital of Chongqing Medical University (awarded to YC).

\section{Authors' contributions}

JPG and MYM designed the study. FCY, YC and $\mathrm{CMH}$ carried out the animal experiments. HW, MHW and HW performed the cell experiments. XLY, HB and JYW conducted the molecular cloning and staining experiments. KX, KWZ and WFZ analyzed the data. FCY and YC wrote the manuscript, HPS revised the manuscript. All authors have read and approved the final version of this manuscript.

\section{Acknowledgements}

Not applicable.

\section{References}

1. Jia C (2011) Advances in the regulation of liver regeneration. Expert Rev Gastroenterol Hepatol 5 (1):105-121. doi:10.1586/egh.10.87

2. Miyajima A, Tanaka M, Itoh T (2014) Stem/progenitor cells in liver development, homeostasis, regeneration, and reprogramming. Cell Stem Cell 14 (5):561-574. doi:10.1016/j.stem.2014.04.010

3. Kogure K, Zhang YQ, Maeshima A, Suzuki K, Kuwano H, Kojima I (2000) The role of activin and transforming growth factor-beta in the regulation of organ mass in the rat liver. Hepatology 31 (4):916-921. doi:10.1053/he.2000.6100

4. Boulton R, Woodman A, Calnan D, Selden C, Tam F, Hodgson H (1997) Nonparenchymal cells from regenerating rat liver generate interleukin-1alpha and -1 beta: a mechanism of negative regulation of hepatocyte proliferation. Hepatology 26 (1):49-58. doi:10.1053/jhep.1997.v26.pm0009214451

5. Jin J, Hong IH, Lewis K, lakova P, Breaux M, Jiang Y, Sullivan E, Jawanmardi N, Timchenko L, Timchenko NA (2015) Cooperation of C/EBP family proteins and chromatin remodeling proteins is essential for termination of liver regeneration. Hepatology 61 (1):315-325. doi:10.1002/hep.27295

6. Lai SS, Zhao DD, Cao P, Lu K, Luo OY, Chen WB, Liu J, Jiang EZ, Yu ZH, Lee G, Li J, Yu DC, Xu XJ, Zhu MS, Gao X, Li CJ, Xue B (2016) PP2Acalpha positively regulates the termination of liver regeneration in mice through the AKT/GSK3beta/Cyclin D1 pathway. J Hepatol 64 (2):352-360. doi:10.1016/j.jhep.2015.09.025

7. Huck I, Gunewardena S, Espanol-Suner R, Willenbring H, Apte U (2019) Hepatocyte Nuclear Factor 4 Alpha Activation Is Essential for Termination of Liver Regeneration in Mice. Hepatology 70 (2):666681. doi:10.1002/hep.30405

8. Chen H, Sun Y, Dong R, Yang S, Pan C, Xiang D, Miao M, Jiao B (2011) Mir-34a is upregulated during liver regeneration in rats and is associated with the suppression of hepatocyte proliferation. PloS one 6 (5):e20238-e20238. doi:10.1371/journal.pone.0020238 
9. Chang TC, Wentzel EA, Kent OA, Ramachandran K, Mullendore M, Lee KH, Feldmann G, Yamakuchi M, Ferlito M, Lowenstein CJ, Arking DE, Beer MA, Maitra A, Mendell JT (2007) Transactivation of miR34 a by p53 broadly influences gene expression and promotes apoptosis. Mol Cell 26 (5):745-752. doi:10.1016/j.molcel.2007.05.010

10. Yamakuchi M, Ferlito M, Lowenstein CJ (2008) miR-34a repression of SIRT1 regulates apoptosis. Proc Natl Acad Sci U S A 105 (36):13421-13426. doi:10.1073/pnas.0801613105

11. Lee J, Padhye A, Sharma A, Song G, Miao J, Mo YY, Wang L, Kemper JK (2010) A pathway involving farnesoid $X$ receptor and small heterodimer partner positively regulates hepatic sirtuin 1 levels via microRNA-34a inhibition. J Biol Chem 285 (17):12604-12611. doi:10.1074/jbc.M109.094524

12. Drudi Metalli V, Mancino MG, Mancino A, Torrice A, Gatto M, Attili AF, Alpini G, Alvaro D (2007) Bile salts regulate proliferation and apoptosis of liver cells by modulating the IGF1 system. Dig Liver Dis 39 (7):654-662. doi:10.1016/j.dld.2007.03.008

13. Fan $M$, Wang $X, X u$ G, Yan Q, Huang W (2015) Bile acid signaling and liver regeneration. Biochim Biophys Acta 1849 (2):196-200. doi:10.1016/j.bbagrm.2014.05.021

14. Monte MJ, El-Mir MY, Sainz GR, Bravo P, Marin JJ (1997) Bile acid secretion during synchronized rat liver regeneration. Biochim Biophys Acta 1362 (1):56-66

15. Ding L, Yang Y, Qu Y, Yang T, Wang K, Liu W, Xia W (2015) Bile acid promotes liver regeneration via farnesoid $X$ receptor signaling pathways in rats. Mol Med Rep 11 (6):4431-4437. doi:10.3892/mmr.2015.3270

16. Hofmann AF, Hagey LR, Krasowski MD (2010) Bile salts of vertebrates: structural variation and possible evolutionary significance. J Lipid Res 51 (2):226-246. doi:10.1194/jlr.R000042

17. Sayin SI, Wahlstrom A, Felin J, Jantti S, Marschall HU, Bamberg K, Angelin B, Hyotylainen T, Oresic M, Backhed $F$ (2013) Gut microbiota regulates bile acid metabolism by reducing the levels of tauro-betamuricholic acid, a naturally occurring FXR antagonist. Cell Metab 17 (2):225-235.

doi:10.1016/j.cmet.2013.01.003

18. Gan L, Liu Z, Sun C (2018) Obesity linking to hepatocellular carcinoma: A global view. Biochim Biophys Acta Rev Cancer 1869 (2):97-102. doi:10.1016/j.bbcan.2017.12.006

19. Olivier M, Hussain SP, Caron de Fromentel C, Hainaut P, Harris CC (2004) TP53 mutation spectra and load: a tool for generating hypotheses on the etiology of cancer. IARC Sci Publ (157):247-270

20. Hussain SP, Schwank J, Staib F, Wang XW, Harris CC (2007) TP53 mutations and hepatocellular carcinoma: insights into the etiology and pathogenesis of liver cancer. Oncogene 26 (15):2166-2176. doi:10.1038/sj.onc.1210279

21. Kanno S-i, Kurauchi K, Tomizawa A, Yomogida S, Ishikawa M (2015) Pifithrin-alpha has a p53independent cytoprotective effect on docosahexaenoic acid-induced cytotoxicity in human hepatocellular carcinoma HepG2 cells. Toxicology Letters 232 (2):393-402. doi:https://doi.org/10.1016/j.toxlet.2014.11.016

22. Wahlstrom A, Sayin SI, Marschall HU, Backhed F (2016) Intestinal Crosstalk between Bile Acids and Microbiota and Its Impact on Host Metabolism. Cell Metab 24 (1):41-50. 
doi:10.1016/j.cmet.2016.05.005

23. de Aguiar Vallim TQ, Tarling EJ, Edwards PA (2013) Pleiotropic roles of bile acids in metabolism. Cell Metab 17 (5):657-669. doi:10.1016/j.cmet.2013.03.013

24. Buryova H, Chalupsky K, Zbodakova O, Kanchev I, Jirouskova M, Gregor M, Sedlacek R (2013) Liver protective effect of ursodeoxycholic acid includes regulation of ADAM17 activity. BMC Gastroenterol 13:155. doi:10.1186/1471-230X-13-155

25. Vogelstein B, Lane D, Levine AJ (2000) Surfing the p53 network. Nature 408 (6810):307-310. doi:10.1038/35042675

26. Hat B, Kochanczyk M, Bogdal MN, Lipniacki T (2016) Feedbacks, Bifurcations, and Cell Fate Decision-Making in the p53 System. PLoS Comput Biol 12 (2):e1004787. doi:10.1371/journal.pcbi.1004787

27. Lin KT, Ma WK, Scharner J, Liu YR, Krainer AR (2018) A human-specific switch of alternatively spliced AFMID isoforms contributes to TP53 mutations and tumor recurrence in hepatocellular carcinoma. Genome Res. doi:10.1101/gr.227181.117

28. Bommer GT, Gerin I, Feng Y, Kaczorowski AJ, Kuick R, Love RE, Zhai Y, Giordano TJ, Qin ZS, Moore BB, MacDougald OA, Cho KR, Fearon ER (2007) p53-mediated activation of miRNA34 candidate tumor-suppressor genes. Curr Biol 17 (15):1298-1307. doi:10.1016/j.cub.2007.06.068

29. He L, He X, Lim LP, de Stanchina E, Xuan Z, Liang Y, Xue W, Zender L, Magnus J, Ridzon D, Jackson AL, Linsley PS, Chen C, Lowe SW, Cleary MA, Hannon GJ (2007) A microRNA component of the p53 tumour suppressor network. Nature 447 (7148):1130-1134. doi:10.1038/nature05939

30. Raver-Shapira N, Marciano E, Meiri E, Spector Y, Rosenfeld N, Moskovits N, Bentwich Z, Oren M (2007) Transcriptional activation of miR-34a contributes to p53-mediated apoptosis. Mol Cell 26 (5):731-743. doi:10.1016/j.molcel.2007.05.017

31. Tarasov V, Jung P, Verdoodt B, Lodygin D, Epanchintsev A, Menssen A, Meister G, Hermeking H (2007) Differential regulation of microRNAs by $p 53$ revealed by massively parallel sequencing: miR-34a is a p53 target that induces apoptosis and G1-arrest. Cell Cycle 6 (13):1586-1593. doi:10.4161/cc.6.13.4436

32. Luo J, Nikolaev AY, Imai S, Chen D, Su F, Shiloh A, Guarente L, Gu W (2001) Negative control of p53 by Sir2alpha promotes cell survival under stress. Cell 107 (2):137-148

33. Vaziri H, Dessain SK, Ng Eaton E, Imai SI, Frye RA, Pandita TK, Guarente L, Weinberg RA (2001) hSIR2(SIRT1) functions as an NAD-dependent p53 deacetylase. Cell 107 (2):149-159

34. Audrito V, Vaisitti T, Rossi D, Gottardi D, D'Arena G, Laurenti L, Gaidano G, Malavasi F, Deaglio S (2011) Nicotinamide blocks proliferation and induces apoptosis of chronic lymphocytic leukemia cells through activation of the p53/miR-34a/SIRT1 tumor suppressor network. Cancer Res 71 (13):4473-4483. doi:10.1158/0008-5472.CAN-10-4452

35. Lou G, Liu Y, Wu S, Xue J, Yang F, Fu H, Zheng M, Chen Z (2015) The p53/miR-34a/SIRT1 Positive Feedback Loop in Quercetin-Induced Apoptosis. Cell Physiol Biochem 35 (6):2192-2202. doi:10.1159/000374024 
36. Ferreira DM, Afonso MB, Rodrigues PM, Simao AL, Pereira DM, Borralho PM, Rodrigues CM, Castro RE (2014) c-Jun N-terminal kinase 1/c-Jun activation of the p53/microRNA 34a/sirtuin 1 pathway contributes to apoptosis induced by deoxycholic acid in rat liver. Mol Cell Biol 34 (6):1100-1120. doi:10.1128/MCB.00420-13

37. Neuveut C, Wei Y, Buendia MA (2010) Mechanisms of HBV-related hepatocarcinogenesis. J Hepatol 52 (4):594-604. doi:10.1016/j.jhep.2009.10.033

38. Kan Z, Zheng H, Liu X, Li S, Barber TD, Gong Z, Gao H, Hao K, Willard MD, Xu J, Hauptschein R, Rejto PA, Fernandez J, Wang G, Zhang Q, Wang B, Chen R, Wang J, Lee NP, Zhou W, Lin Z, Peng Z, Yi K, Chen S, Li L, Fan X, Yang J, Ye R, Ju J, Wang K, Estrella H, Deng S, Wei P, Qiu M, Wulur IH, Liu J, Ehsani ME, Zhang C, Loboda A, Sung WK, Aggarwal A, Poon RT, Fan ST, Wang J, Hardwick J, Reinhard C, Dai H, Li Y, Luk JM, Mao M (2013) Whole-genome sequencing identifies recurrent mutations in hepatocellular carcinoma. Genome Res 23 (9):1422-1433. doi:10.1101/gr.154492.113

39. Xia C, Shui L, Lou G, Ye B, Zhu W, Wang J, Wu S, Xu X, Mao L, Xu W, Chen Z, Liu Y, Zheng M (2017) 0404 inhibits hepatocellular carcinoma through a p53/miR-34a/SIRT1 positive feedback loop. Sci Rep 7 (1):4396. doi:10.1038/s41598-017-04487-x

\section{Figures}




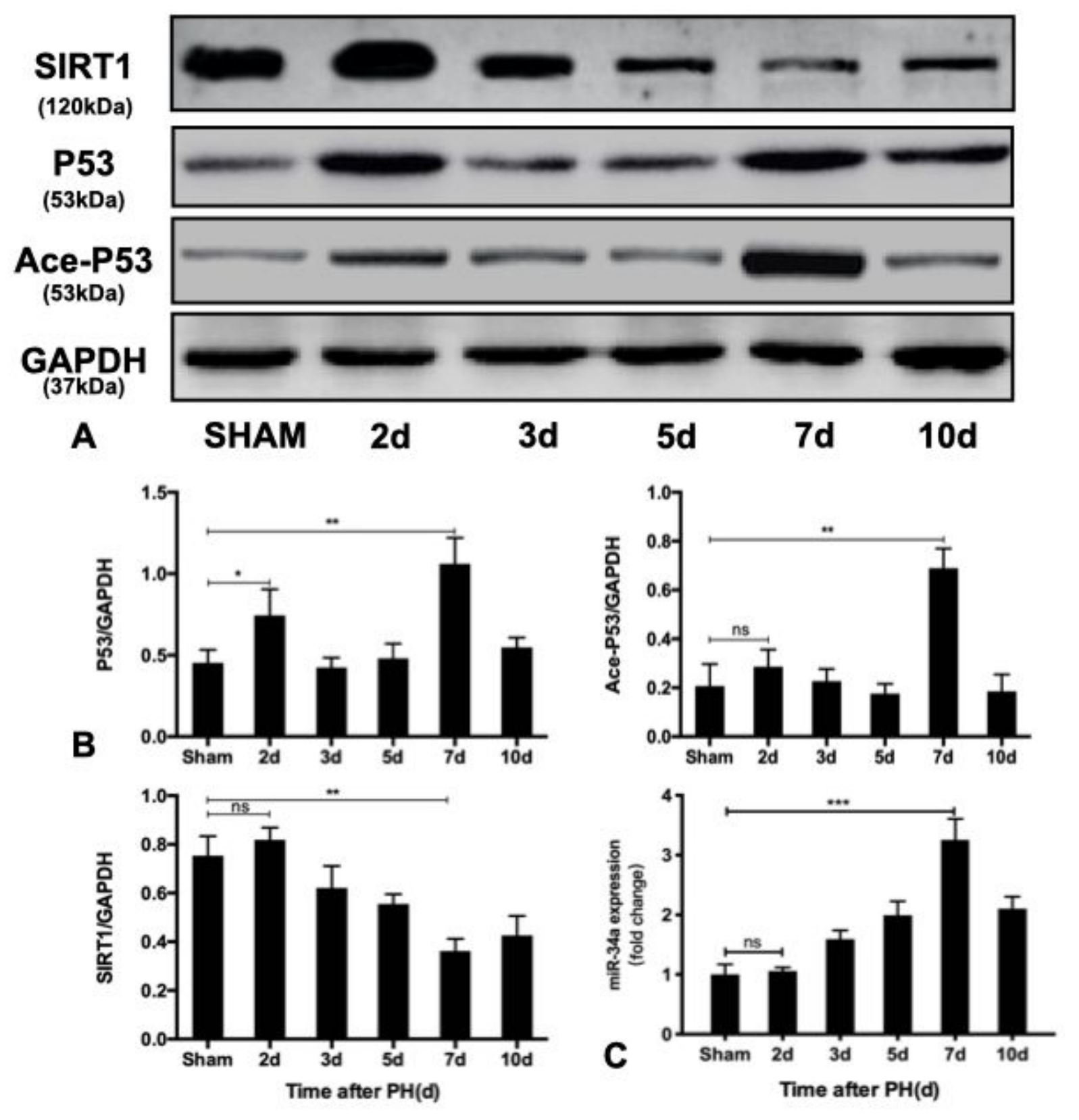

Figure 1

P53/miR-34a/SIRT1 positive feedback loop expression during LR. (A) Protein expression of the P53/miR34a/SIRT1 positive feedback loop during LR progression was analyzed via western blot. (B) Quantification of hepatic P53, Ace-P53, and SIRT1 expression at the indicated time points after PH by WB. (C) Quantification of hepatic miR-34a expression at the indicated time points after PH by qPCR. (ns: not significant, $\left.p>0.05 ;{ }^{*}, p<0.05 ; * \star, p<0.01 ; * \star \star, p<0.001\right)$. 


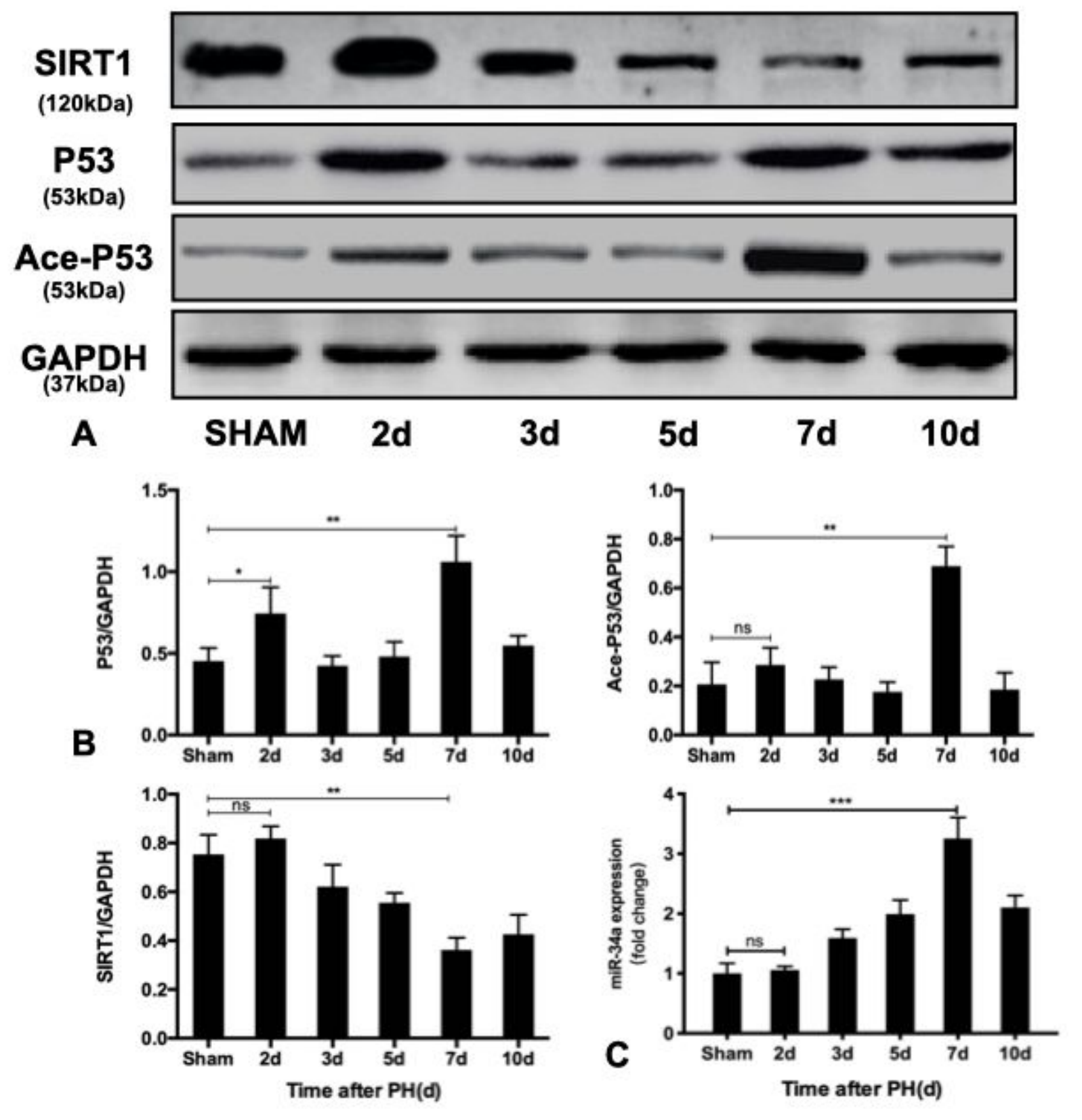

Figure 1

P53/miR-34a/SIRT1 positive feedback loop expression during LR. (A) Protein expression of the P53/miR34a/SIRT1 positive feedback loop during LR progression was analyzed via western blot. (B) Quantification of hepatic P53, Ace-P53, and SIRT1 expression at the indicated time points after PH by WB. (C) Quantification of hepatic miR-34a expression at the indicated time points after PH by qPCR. (ns: not significant, $\left.p>0.05 ;{ }^{*}, p<0.05 ; * \star, p<0.01 ; * \star \star, p<0.001\right)$. 

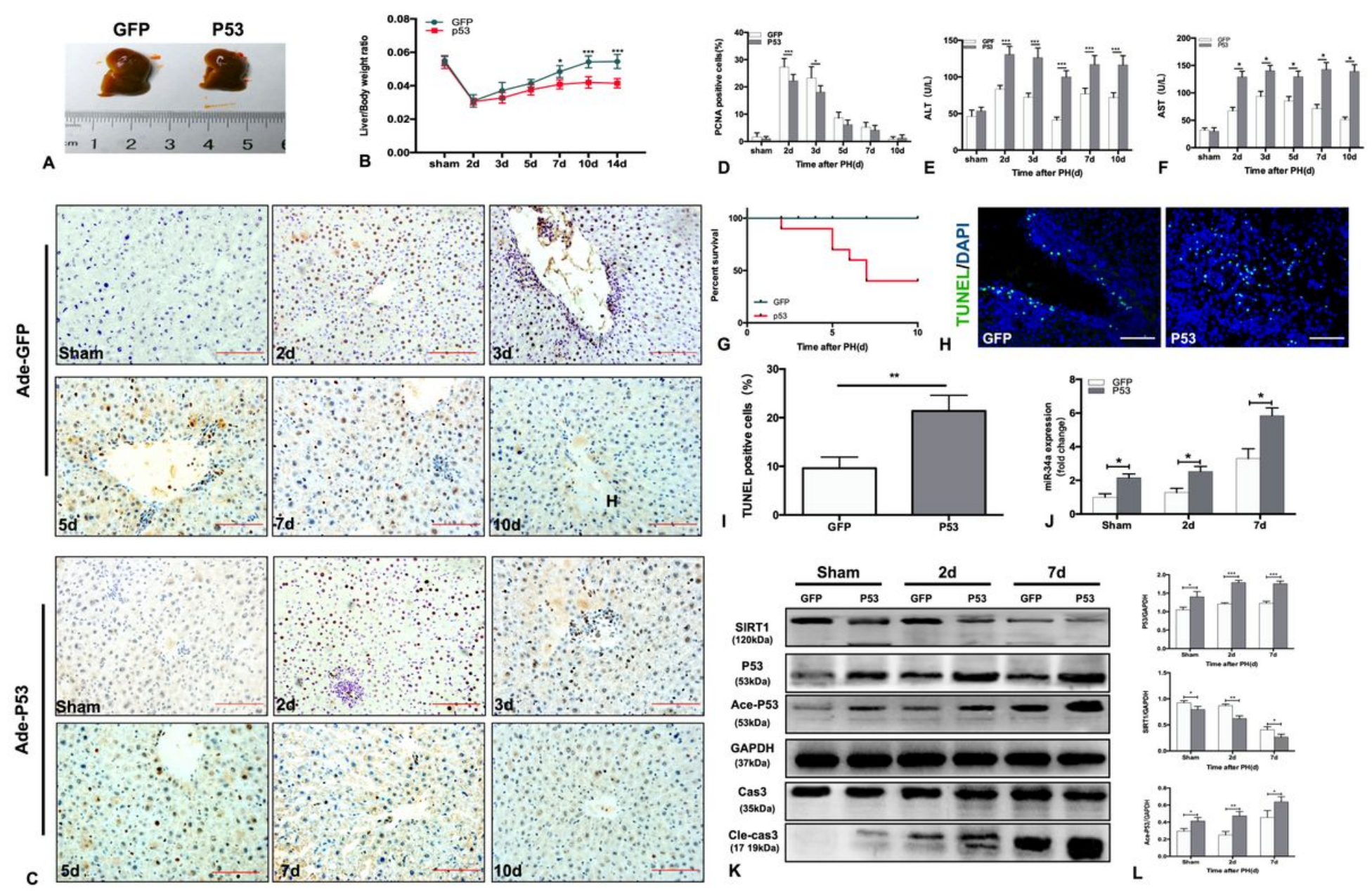

\section{Figure 2}

Overexpression of wild-type P53 terminates LR and activates the P53/miR-34a/SIRT1 positive feedback loop early during LR. (A) Representative livers from mice at 7 days after PH between Ade-GFP and AdeP53 mice. (B) Liver weight relative to body weight at the indicated time points after PH. (C) Representative images of PCNA staining at the indicated time points after PH from the Ade-GFP and Ade-P53 groups (magnification: $\times 200$, scale bars represent $50 \mu \mathrm{m}$ ). (D) Quantification of PCNA-positive cells in the liver at the indicated time points after PH. (E, F) Serum AST and ALT levels in Ade-GFP and Ade-P53 mice after $\mathrm{PH}$. (G) Survival rate of mice that underwent PH from the Ade-GFP and Ade-P53 groups. $(\mathrm{H})$ Representative images of TUNEL staining at day 7 after $\mathrm{PH}$ in liver tissue (magnification: $\times 400$ ). (I)Quantification of TUNEL-positive cells in the liver at day 7 after PH. (J) Quantification of hepatic miR34a expression between Ade-GFP and Ade-P53 mice at the indicated time points after PH by qPCR. (K) Protein expression of P53/miR-34a/SIRT1 positive feedback loop genes between Ade-GFP and Ade-P53 mice at the indicated time points after PH. (L) Quantification of hepatic P53, Ace-P53, and SIRT1 protein expression by WB. $\left({ }^{*}, p<0.05 ; * \star, p<0.01 ; * \star *, p<0.001\right)$. 

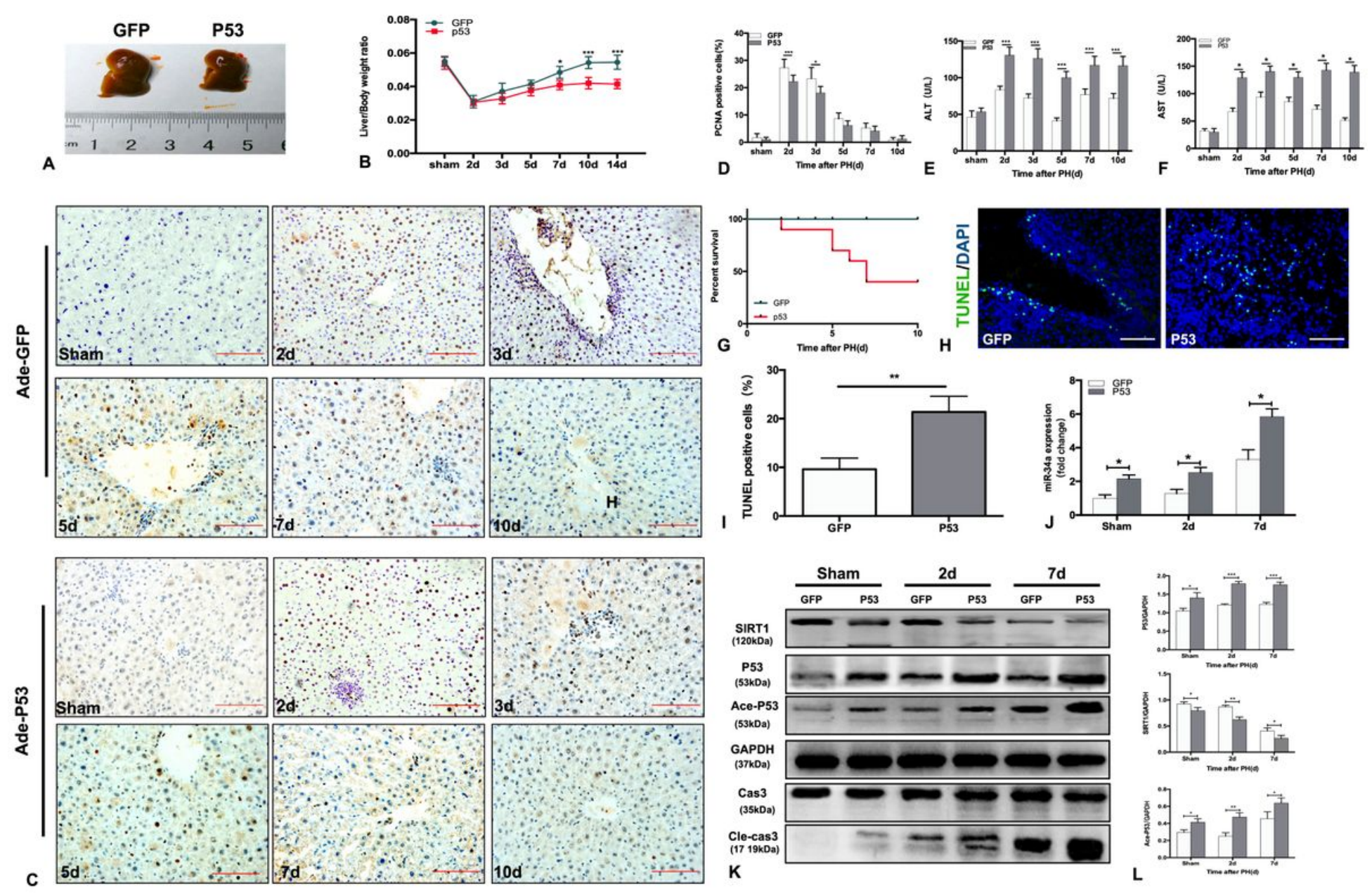

\section{Figure 2}

Overexpression of wild-type P53 terminates LR and activates the P53/miR-34a/SIRT1 positive feedback loop early during LR. (A) Representative livers from mice at 7 days after PH between Ade-GFP and AdeP53 mice. (B) Liver weight relative to body weight at the indicated time points after $\mathrm{PH}$. (C) Representative images of PCNA staining at the indicated time points after PH from the Ade-GFP and Ade-P53 groups (magnification: $\times 200$, scale bars represent $50 \mu \mathrm{m}$ ). (D) Quantification of PCNA-positive cells in the liver at the indicated time points after PH. (E, F) Serum AST and ALT levels in Ade-GFP and Ade-P53 mice after $\mathrm{PH}$. (G) Survival rate of mice that underwent PH from the Ade-GFP and Ade-P53 groups. $(\mathrm{H})$ Representative images of TUNEL staining at day 7 after $\mathrm{PH}$ in liver tissue (magnification: $\times 400$ ). (I)Quantification of TUNEL-positive cells in the liver at day 7 after PH. (J) Quantification of hepatic miR34a expression between Ade-GFP and Ade-P53 mice at the indicated time points after PH by qPCR. (K) Protein expression of P53/miR-34a/SIRT1 positive feedback loop genes between Ade-GFP and Ade-P53 mice at the indicated time points after PH. (L) Quantification of hepatic P53, Ace-P53, and SIRT1 protein expression by WB. $\left({ }^{*}, p<0.05 ; * \star, p<0.01 ; * \star *, p<0.001\right)$. 


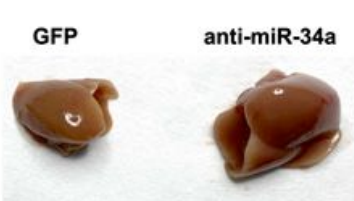

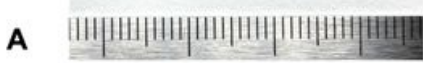

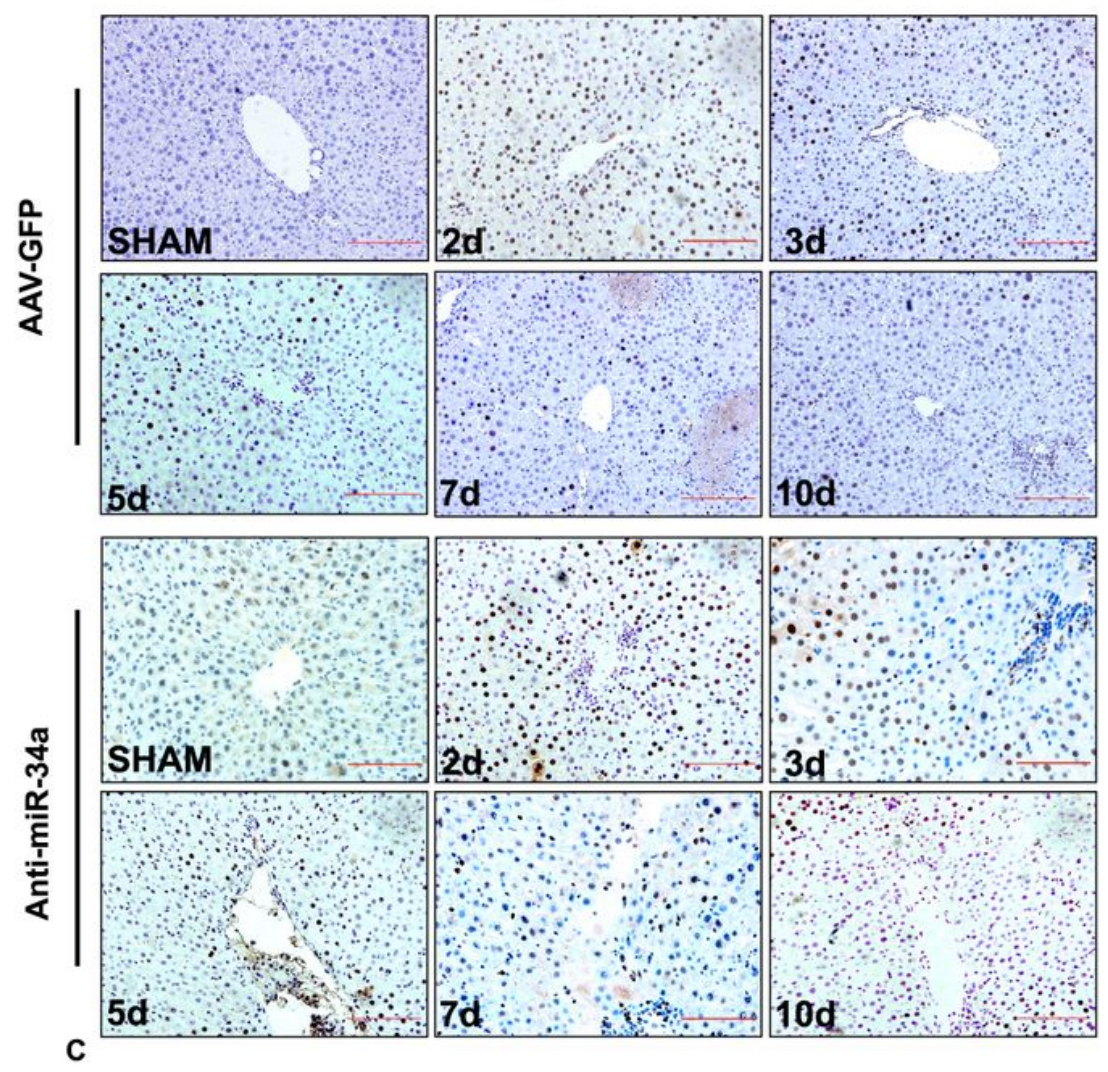

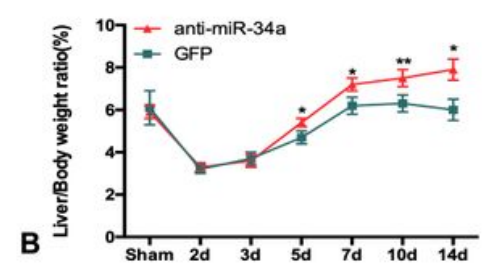

$10 d$


G

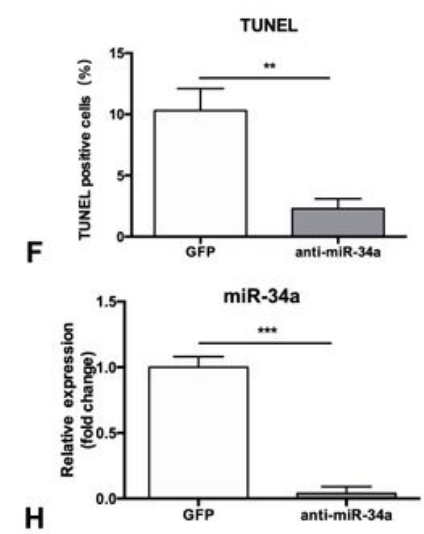

Figure 3

Knock-down of miR-34a suppresses the P53/miR-34a/SIRT1 positive feedback loop early during LR. (A) Representative livers from mice at 7 days after PH between AAV-GFP and AAV-anti-miR-34a mice. (B) Liver weight relative to body weight at the indicated time points after $\mathrm{PH}$. (C)Representative images of PCNA staining at the indicated time points after PH in the GFP and anti-miR-34a groups (magnification: $\times 200$, scale bars represent $50 \mu \mathrm{m}$ ).(D) Quantification of PCNA-positive cells in the liver at the indicated time points after $\mathrm{PH}$.(E) Representative images of TUNEL staining at day 7 after $\mathrm{PH}$ in liver tissue(magnification: $\times 400)$. (F)Quantification of TUNEL-positive cells in the liver at day 7 after PH. (G)Protein expression of P53/miR-34a/SIRT1 positive feedback loop genes between AAV-GFP and AAVmiR-34a mice at day 7 after PH. (H) Quantification of hepatic miR-34a expression between AAV-GFP and AAV-miR-34a mice at day 7 after PH by qPCR. (*, $p<0.05 ; * \star, p<0.01 ; * \star *, p<0.001)$. 


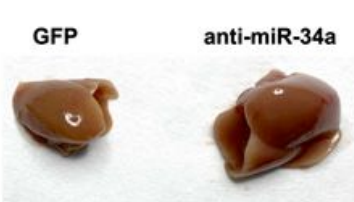

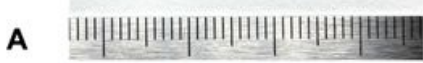

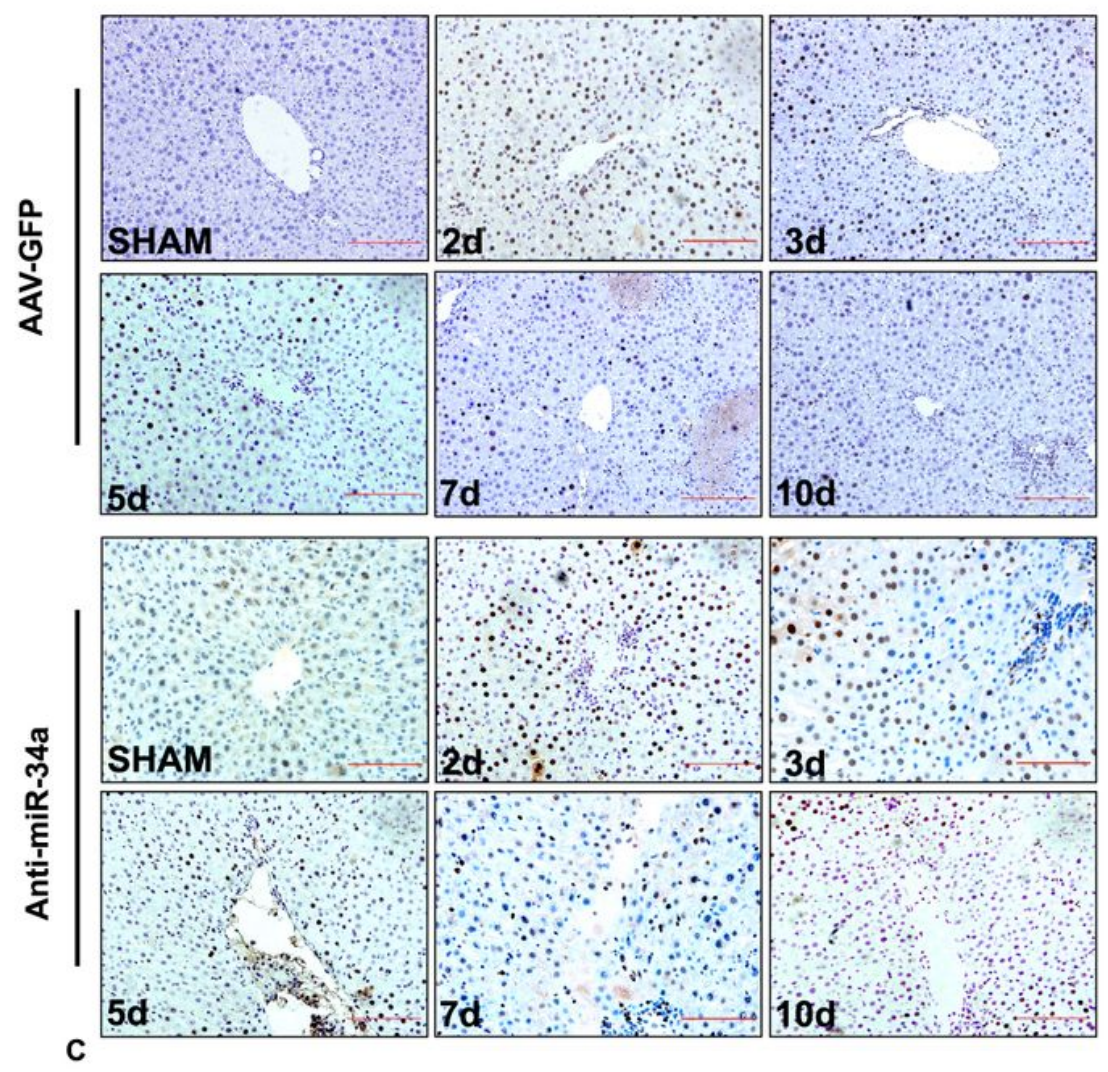

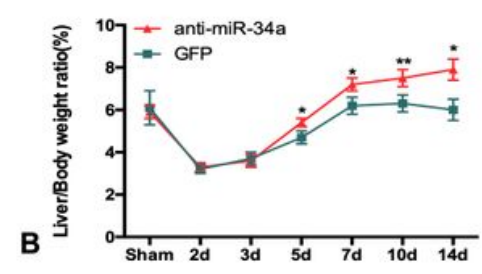

$10 d$
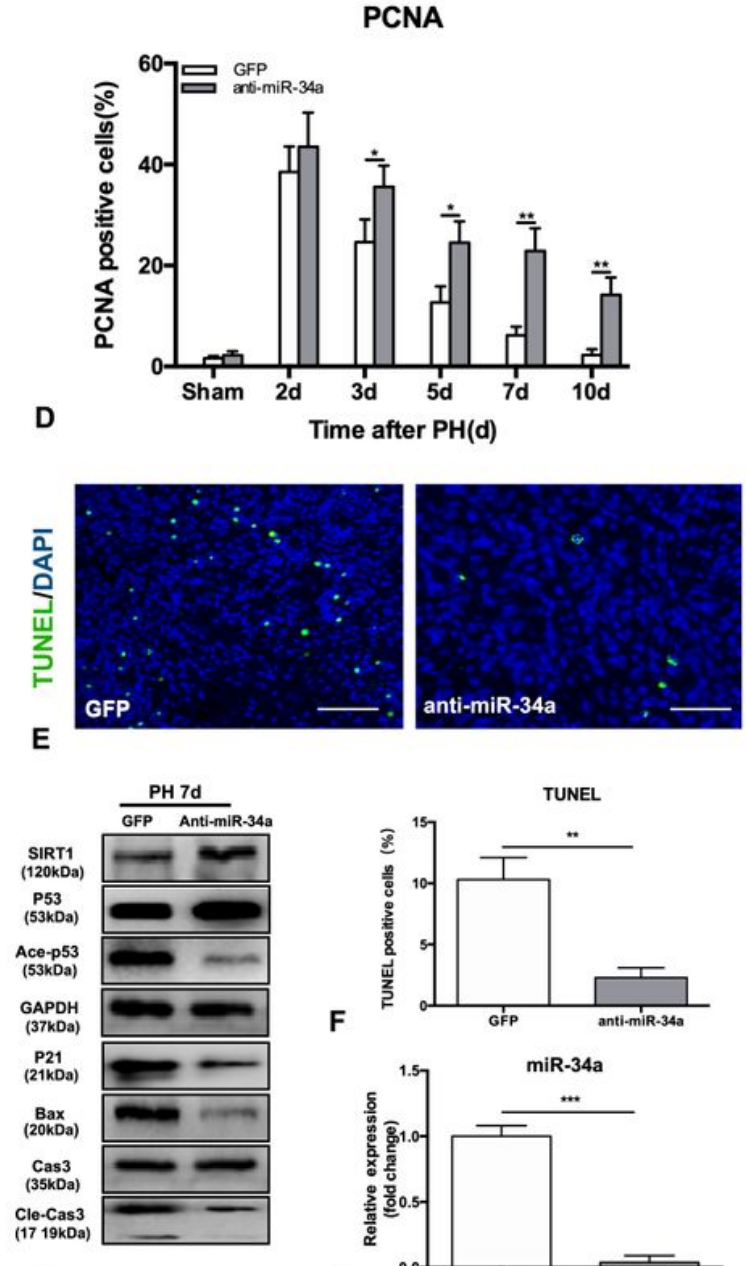

G

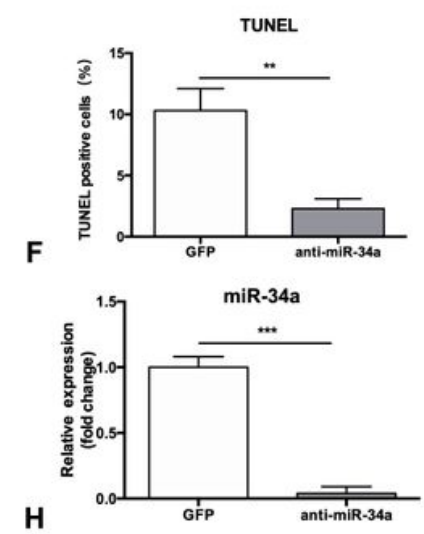

Figure 3

Knock-down of miR-34a suppresses the P53/miR-34a/SIRT1 positive feedback loop early during LR. (A) Representative livers from mice at 7 days after PH between AAV-GFP and AAV-anti-miR-34a mice. (B) Liver weight relative to body weight at the indicated time points after $\mathrm{PH}$. (C)Representative images of PCNA staining at the indicated time points after PH in the GFP and anti-miR-34a groups (magnification: $\times 200$, scale bars represent $50 \mu \mathrm{m}$ ).(D) Quantification of PCNA-positive cells in the liver at the indicated time points after $\mathrm{PH}$.(E) Representative images of TUNEL staining at day 7 after $\mathrm{PH}$ in liver tissue(magnification: $\times 400)$. (F)Quantification of TUNEL-positive cells in the liver at day 7 after PH. (G)Protein expression of P53/miR-34a/SIRT1 positive feedback loop genes between AAV-GFP and AAVmiR-34a mice at day 7 after PH. (H) Quantification of hepatic miR-34a expression between AAV-GFP and AAV-miR-34a mice at day 7 after PH by qPCR. (*, $p<0.05 ; * \star, p<0.01 ; * \star *, p<0.001)$. 

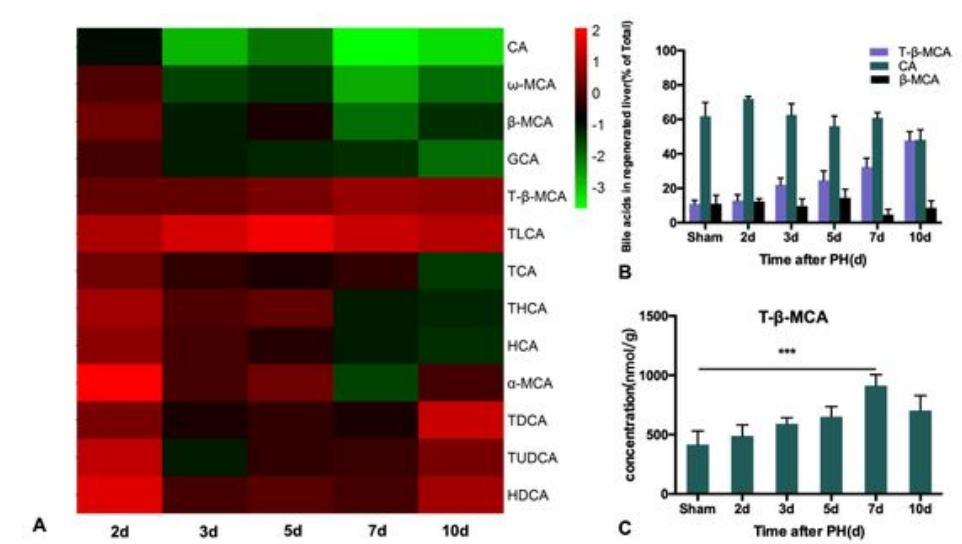

NC GFP



D
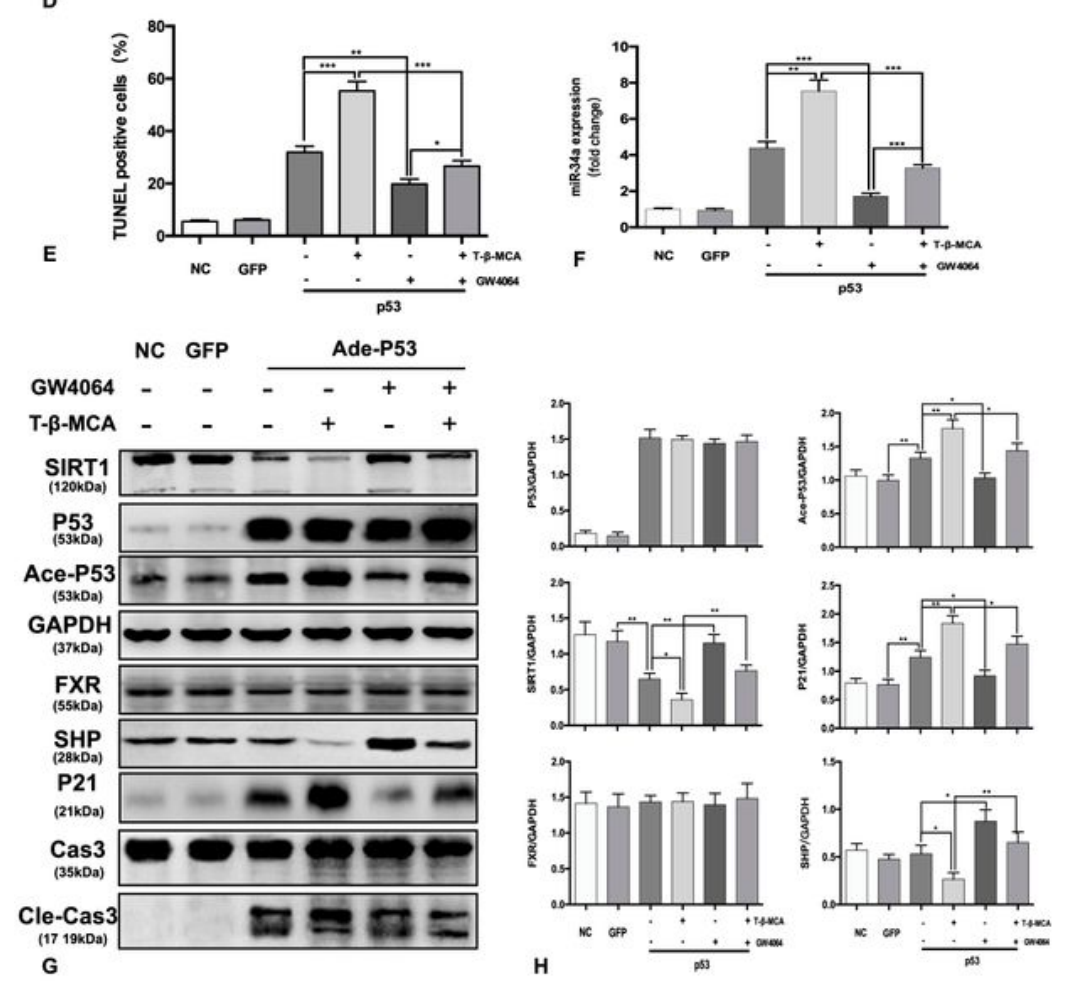

\section{Figure 4}

T- $\beta$-MCA enhanced the proapoptotic effect of the P53-activated P53/miR-34a/SIRT1 positive feedback loop by suppressing the FXR/SHP signaling pathway in vitro. (A) Heatmap of changed Bas during LR. (B) Percentage of the top 3 BAs at the indicated time points after $\mathrm{PH}$. (C) Concentration of T- $\beta$-MCA at the indicated time points after PH. (D)TUNEL staining of primary hepatocytes after knock-in of P53 with/without administration of T- $\beta-M C A$ and GW4064(magnification: $\times 400$, Scale bars represent $50 \mu \mathrm{m}$ ). 
(E)Quantification of TUNEL-positive cells after knock-in of P53 with/without administration of T- $\beta$-MCA and GW4064.(F)Quantification of hepatic miR-34a expression in mouse primary hepatocytes after knockin of P53 with/without administration of T- $\beta-M C A$ and GW4064 by qPCR.(G)Protein expression of P53/miR-34a/SIRT1 positive feedback loop genes and FXR/SHP signaling after knock-in of P53 with/without administration of T- $\beta-M C A$ and GW4064. Cells were pretreated with/without $100 \mu \mathrm{M} \mathrm{T-} \beta$ MCA or $1 \mu \mathrm{M}$ GW4064 for $12 \mathrm{~h}$. (H) Quantification of P53, Ace-P53, SIRT1, P21, FXR, and SHP protein expression by WB. (ns: $p>0.05 ; *, p<0.05 ; * \star, p<0.01 ; * * *, p<0.001)$.

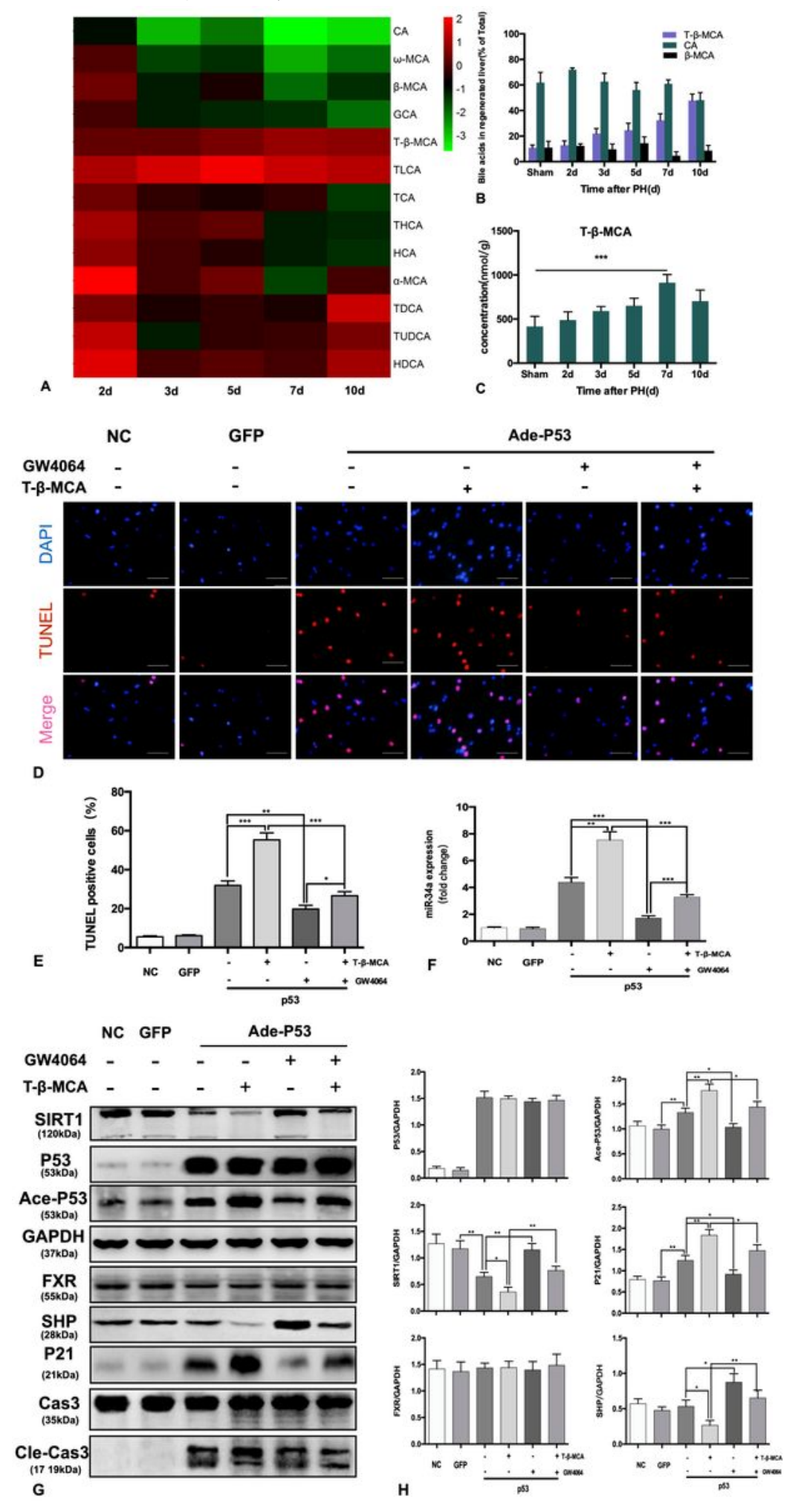




\section{Figure 4}

T- $\beta$-MCA enhanced the proapoptotic effect of the P53-activated P53/miR-34a/SIRT1 positive feedback loop by suppressing the FXR/SHP signaling pathway in vitro. (A) Heatmap of changed Bas during LR. (B) Percentage of the top 3 BAs at the indicated time points after $\mathrm{PH}$. (C) Concentration of T- $\beta$-MCA at the indicated time points after PH. (D)TUNEL staining of primary hepatocytes after knock-in of P53 with/without administration of T- $\beta$-MCA and GW4064(magnification: $\times 400$, Scale bars represent $50 \mu \mathrm{m}$ ). (E)Quantification of TUNEL-positive cells after knock-in of P53 with/without administration of T- $\beta$-MCA and GW4064.(F)Quantification of hepatic miR-34a expression in mouse primary hepatocytes after knockin of P53 with/without administration of T- $\beta-M C A$ and GW4064 by qPCR.(G)Protein expression of P53/miR-34a/SIRT1 positive feedback loop genes and FXR/SHP signaling after knock-in of P53 with/without administration of T- $\beta$-MCA and GW4064. Cells were pretreated with/without $100 \mu \mathrm{M}$ T- $\beta$ MCA or $1 \mu \mathrm{M}$ GW4064 for $12 \mathrm{~h}$. (H) Quantification of P53, Ace-P53, SIRT1, P21, FXR, and SHP protein expression by WB. (ns: $p>0.05 ; *, p<0.05 ; * \star, p<0.01 ; * \star *, p<0.001)$. 

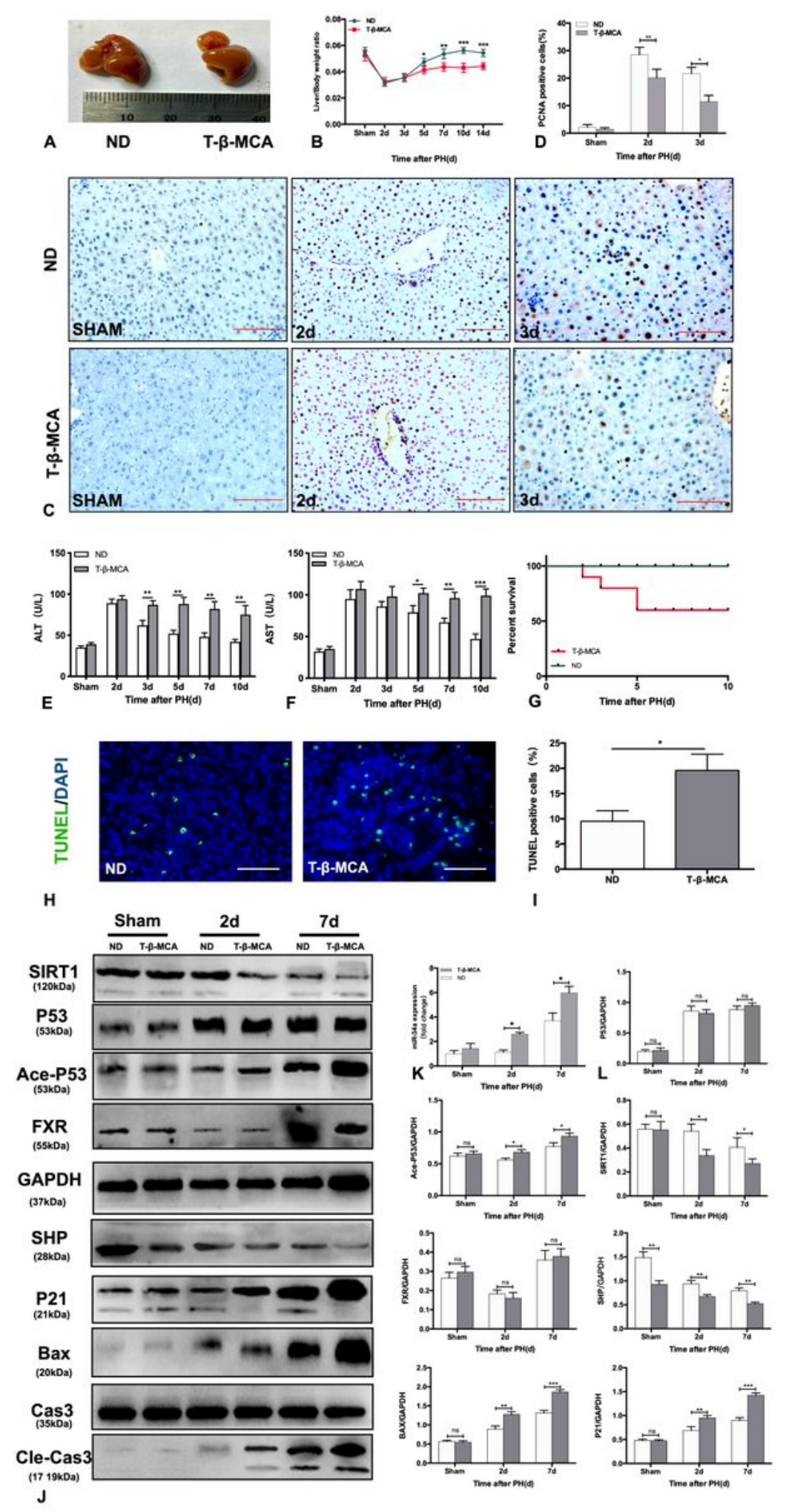

Figure 5

T- $\beta$-MCA facilitates the P53/miR-34a/SIRT1 positive feedback loop during LR by suppressing the FXR/SHP signaling pathway in vivo. (A) Representative livers from mice at 7 days after PH between ND and T- $\beta-M C A$ mice. Mice in the T- $\beta$-MCA group were administered T- $\beta-M C A(400 \mathrm{mg} / \mathrm{kg}$ ) by gavage 1 day before $\mathrm{PH}$ and every 3 days after $\mathrm{PH}(\mathrm{B})$ Liver weight relative to body weight at the indicated time points after PH.(C) Representative images of PCNA staining at the indicated time points after PH from the ND 
and T- $\beta$-MCA groups. (magnification: $\times 200$, Scale bars represent $50 \mu \mathrm{m}$ ). (D) Quantification of PCNApositive cells in the liver at the indicated time points after $\mathrm{PH}$. (E, F) Serum AST and ALT levels in mice from the ND and MCA groups after $\mathrm{PH}$. (G) Survival rate of mice that underwent PH from the ND and T- $\beta$ MCA groups. $(\mathrm{H})$ Representative images of TUNEL staining at day 7 after $\mathrm{PH}$ in liver tissue (magnification: $\times 400$ ). (I)Quantification of TUNEL-positive cells in the liver at day 7 after PH. (J) Protein expression of P53/miR-34a/SIRT1 positive feedback loop genes and FXR/SHP signaling with/without administration of T- $\beta$-MCA. (K)Quantification of hepatic miR-34a expression in mice with/without administration of T- $\beta$-MCA. *, $p<0.05$. (L) Quantification of P53, Ace-P53, SIRT1, P21, BAX, FXR, and SHP protein expression by WB. (ns: not significant, $p>0.05 ;{ }^{*}, p<0.05 ; * \star, p<0.01 ; * \star *, p<0.001$ ). 

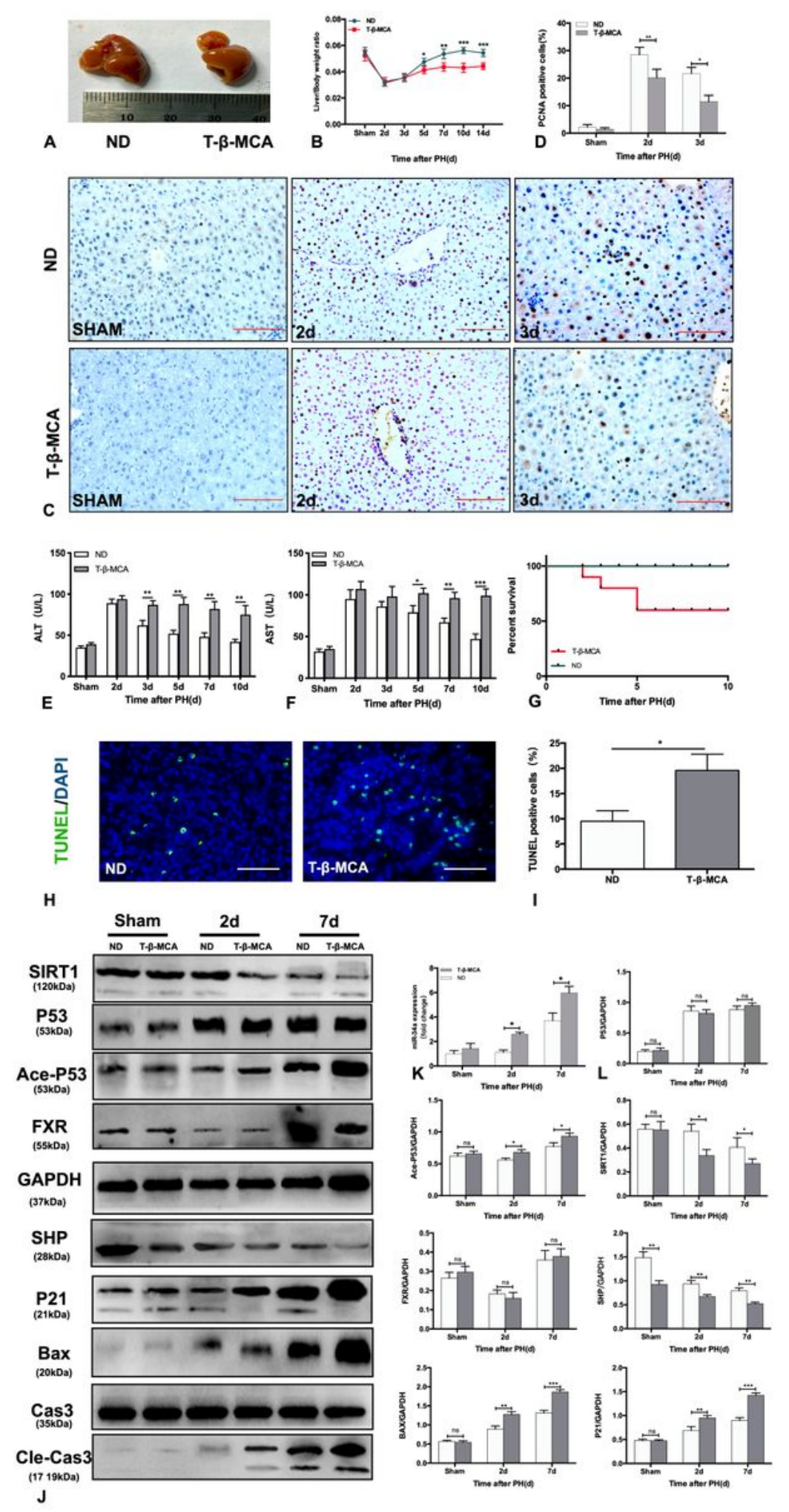

Figure 5

T- $\beta$-MCA facilitates the P53/miR-34a/SIRT1 positive feedback loop during LR by suppressing the FXR/SHP signaling pathway in vivo. (A) Representative livers from mice at 7 days after PH between ND and T- $\beta-M C A$ mice. Mice in the T- $\beta$-MCA group were administered T- $\beta-M C A(400 \mathrm{mg} / \mathrm{kg}$ ) by gavage 1 day before $\mathrm{PH}$ and every 3 days after $\mathrm{PH}(\mathrm{B})$ Liver weight relative to body weight at the indicated time points after PH.(C) Representative images of PCNA staining at the indicated time points after PH from the ND 
and T- $\beta$-MCA groups. (magnification: $\times 200$, Scale bars represent $50 \mu \mathrm{m}$ ). (D) Quantification of PCNApositive cells in the liver at the indicated time points after $\mathrm{PH}$. (E, F) Serum AST and ALT levels in mice from the ND and MCA groups after $\mathrm{PH}$. (G) Survival rate of mice that underwent PH from the ND and T- $\beta$ MCA groups. $(\mathrm{H})$ Representative images of TUNEL staining at day 7 after $\mathrm{PH}$ in liver tissue (magnification: $\times 400$ ). (I)Quantification of TUNEL-positive cells in the liver at day 7 after PH. (J) Protein expression of P53/miR-34a/SIRT1 positive feedback loop genes and FXR/SHP signaling with/without administration of T- $\beta$-MCA. (K)Quantification of hepatic miR-34a expression in mice with/without administration of T- $\beta$-MCA. *, $p<0.05$. (L) Quantification of P53, Ace-P53, SIRT1, P21, BAX, FXR, and SHP protein expression by WB. (ns: not significant, $p>0.05 ;{ }^{*}, p<0.05 ; * \star, p<0.01 ; * \star *, p<0.001$ ). 



Figure 6

Knock-in of wild-type P53 reinforce the P53/miR-34a/SIRT1 positive feedback loop in P53-wild-type HepG2 cell lines. (A) Protein expression of P53/miR-34a/SIRT1 positive feedback loop genes and FXR/SHP signaling after knock-in of wild-type P53 with/without administration of UDCA in P53-wild-type HepG2 cells. Cells were pretreated with/without $200 \mu \mathrm{M}$ UDCA for $2 \mathrm{~h}$ before the experiment. (B) Quantification of P53, Ace-P53, SIRT1, P21, BAX, FXR, and SHP protein expression by WB. (C) 
Quantification of miR-34a expression in P53-wild-type HepG2 cells after knock-in of wild-type P53 with/without administration of UDCA. (D) TUNEL and EdU staining of P53-wild-type HepG2 cells after knock-in of wild-type P53 with/without administration of UDCA. (E) Quantification of TUNEL-positive and EdU-positive HepG2 cells after knock-in of wild-type P53 with/without administration of UDCA. (ns: not significant, $p>0.05 ; *, p<0.05 ; * \star, p<0.01 ; * \star *, p<0.001)$.
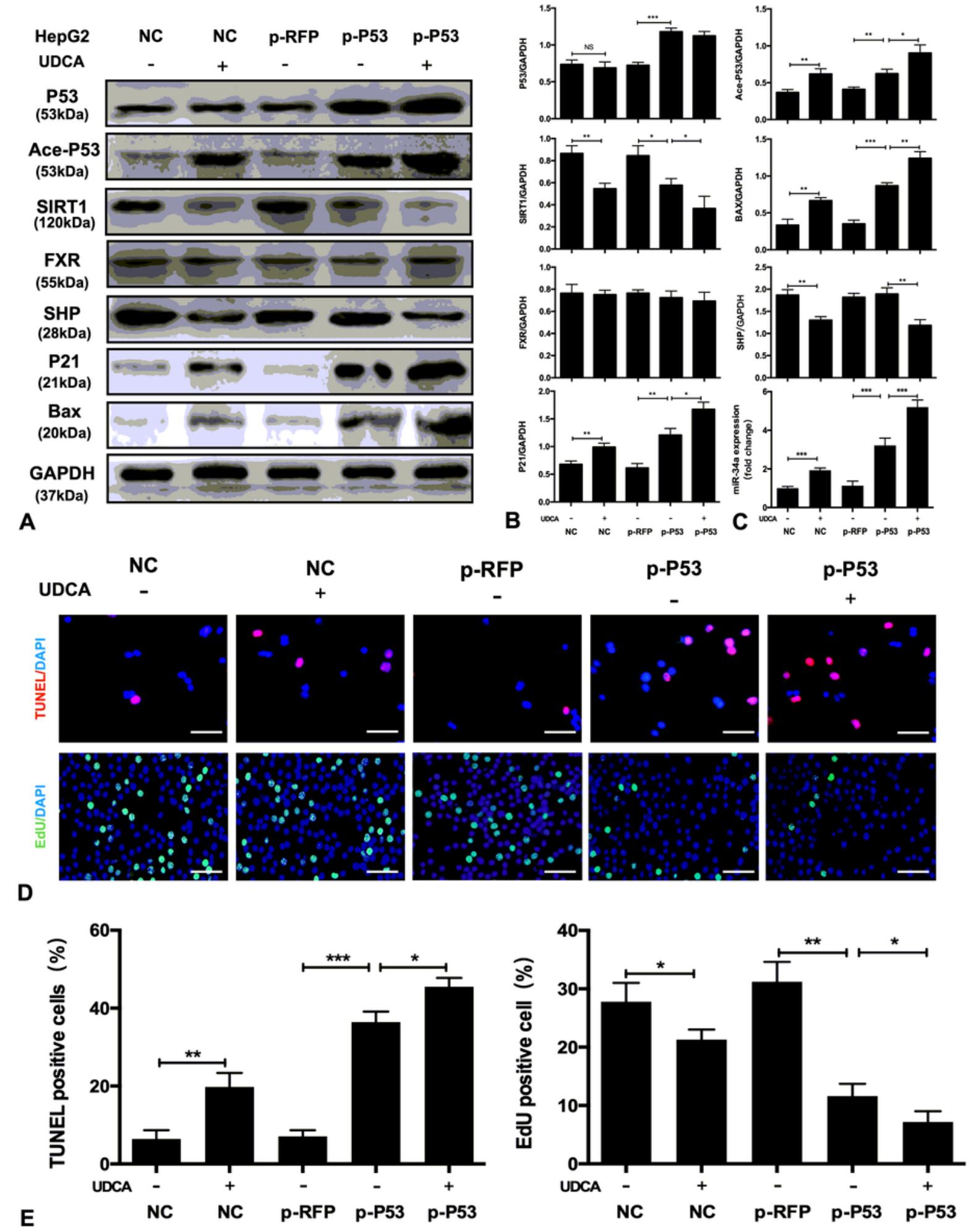

Figure 6 
Knock-in of wild-type P53 reinforce the P53/miR-34a/SIRT1 positive feedback loop in P53-wild-type HepG2 cell lines. (A) Protein expression of P53/miR-34a/SIRT1 positive feedback loop genes and FXR/SHP signaling after knock-in of wild-type P53 with/without administration of UDCA in P53-wild-type HepG2 cells. Cells were pretreated with/without $200 \mu \mathrm{M}$ UDCA for $2 \mathrm{~h}$ before the experiment. (B) Quantification of P53, Ace-P53, SIRT1, P21, BAX, FXR, and SHP protein expression by WB. (C) Quantification of miR-34a expression in P53-wild-type HepG2 cells after knock-in of wild-type P53 with/without administration of UDCA. (D) TUNEL and EdU staining of P53-wild-type HepG2 cells after knock-in of wild-type P53 with/without administration of UDCA. (E) Quantification of TUNEL-positive and EdU-positive HepG2 cells after knock-in of wild-type P53 with/without administration of UDCA. (ns: not significant, $p>0.05 ;{ }^{*}, p<0.05 ; * \star, p<0.01 ; * * *, p<0.001$ ). 

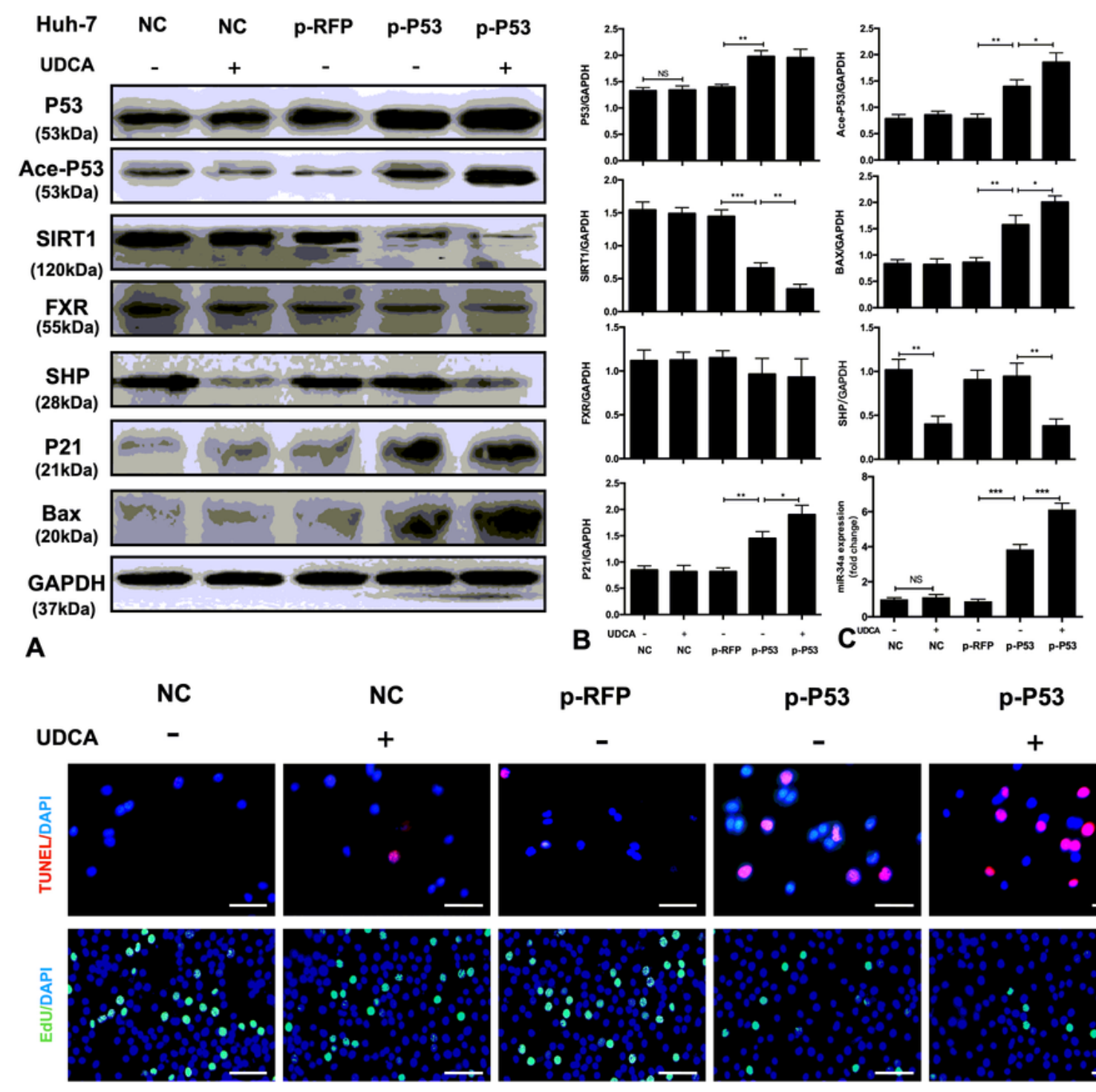

p-RFP

p-P53

p-P53

D
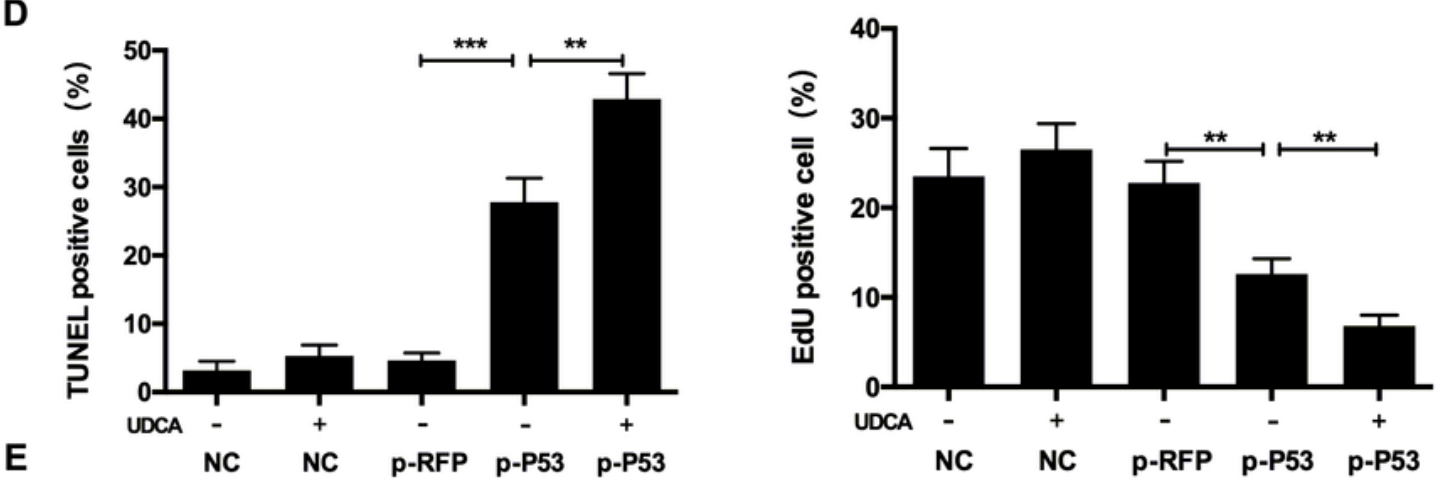

Figure 7

Knock-in of wild-type P53 restored the P53/miR-34a/SIRT1 positive feedback in P53-mutated Huh-7 cell line.(A) Protein expression of P53/miR-34a/SIRT1 positive feedback loop genes and FXR/SHP signaling after knock-in of wild-type P53 with/without administration of UDCA in P53-mutated Huh-7 cells. Cells were pretreated with/without $200 \mu \mathrm{M}$ UDCA for $2 \mathrm{~h}$ before the experiment. (B) Quantification of P53, AceP53, SIRT1, P21, BAX, FXR, and SHP protein expression by WB. (C) Quantification of miR-34a expression 
in P53-mutated Huh-7 cells after knock-in of wild-type P53 with/without administration of UDCA. (D) TUNEL and EdU staining of P53-mutated Huh-7 cells after knock-in of wild-type P53 with/without administration of UDCA (magnification: $\times 400$, Scale bars represent $50 \mu \mathrm{m}$ ). (E) Quantification of TUNELpositive and EdU-positive Huh-7 cells after knock-in of wild-type P53 with/without administration of UDCA. (ns: not significant, $p>0.05 ; *, p<0.05 ; * *, p<0.01 ; * * *, p<0.001$ ).


A
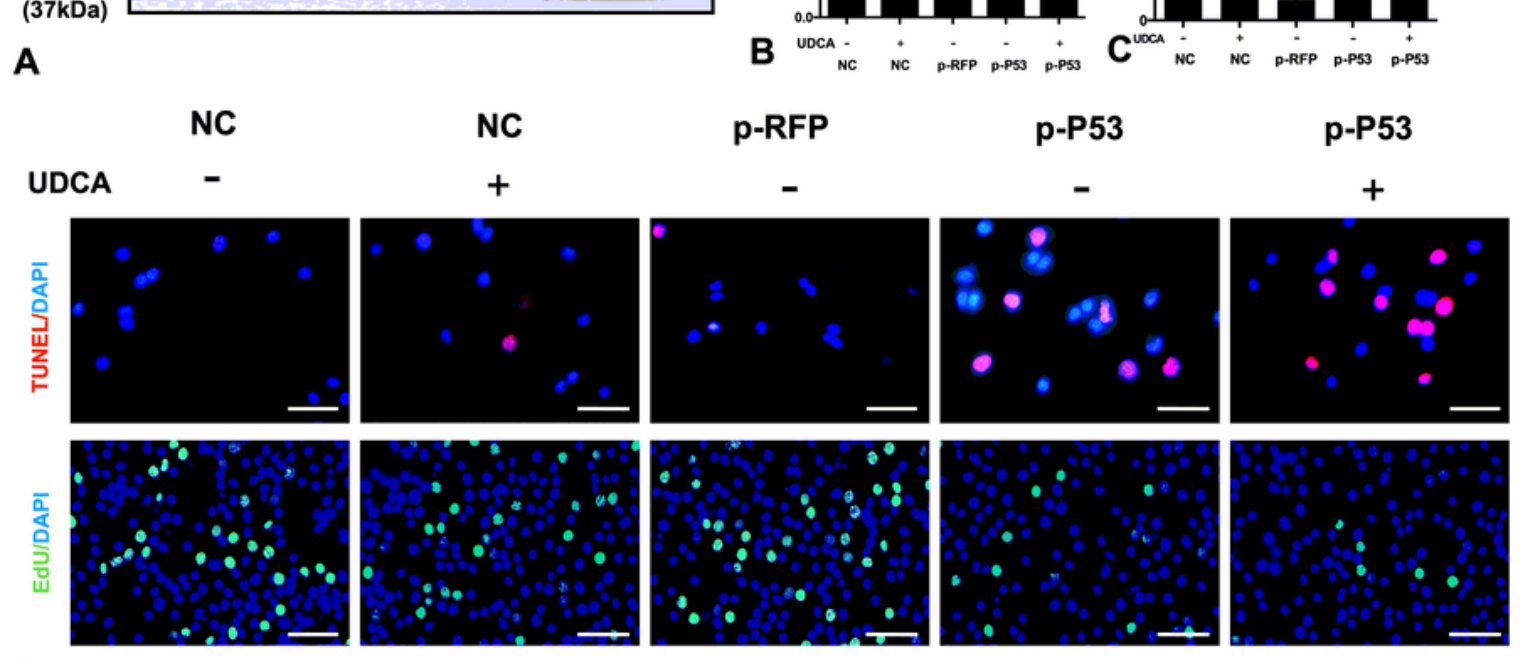

D


Figure 7 
Knock-in of wild-type P53 restored the P53/miR-34a/SIRT1 positive feedback in P53-mutated Huh-7 cell line.(A) Protein expression of P53/miR-34a/SIRT1 positive feedback loop genes and FXR/SHP signaling after knock-in of wild-type P53 with/without administration of UDCA in P53-mutated Huh-7 cells. Cells were pretreated with/without $200 \mu \mathrm{M}$ UDCA for $2 \mathrm{~h}$ before the experiment. (B) Quantification of P53, AceP53, SIRT1, P21, BAX, FXR, and SHP protein expression by WB. (C) Quantification of miR-34a expression in P53-mutated Huh-7 cells after knock-in of wild-type P53 with/without administration of UDCA. (D) TUNEL and EdU staining of P53-mutated Huh-7 cells after knock-in of wild-type P53 with/without administration of UDCA (magnification: $\times 400$, Scale bars represent $50 \mu \mathrm{m}$ ). (E) Quantification of TUNELpositive and EdU-positive Huh-7 cells after knock-in of wild-type P53 with/without administration of UDCA. (ns: not significant, $p>0.05 ; *, p<0.05 ; * \star, p<0.01 ; * \star \star, p<0.001$ ). 

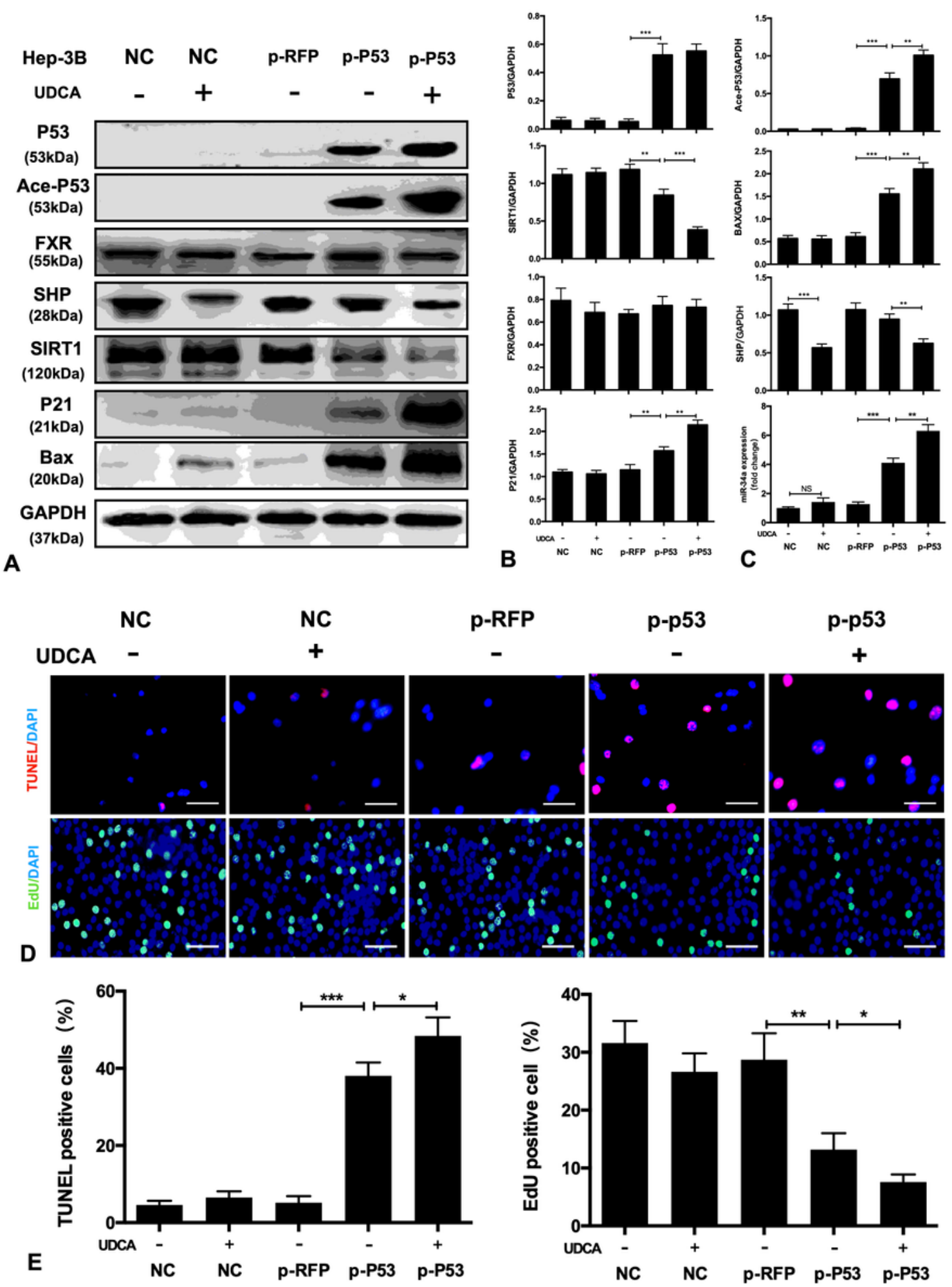

Figure 8

Knock-in of wild-type P53 restored the P53/miR-34a/SIRT1 positive feedback in P53-deficient Hep-3B cell lines. (A) Protein expression of P53/miR-34a/SIRT1 positive feedback loop genes and FXR/SHP signaling after knock-in of wild-type P53 with/without administration of UDCA in P53-deficient Hep3B cells. Cells were pretreated with/without $200 \mu \mathrm{M}$ UDCA for $2 \mathrm{~h}$ before the experiment. (B) Quantification of P53, AceP53, SIRT1, P21, BAX, FXR, and SHP protein expression by WB (C) Quantification of miR-34a expression 
in P53-deficient Hep3B cells after knock-in of wild-type P53 with/without administration of UDCA. (D) TUNEL and EdU staining of P53-deficient Hep3B cells after knock-in of wild-type P53 with/without administration of UDCA (magnification: $\times 400$, Scale bars represent $50 \mu \mathrm{m}$ ). (E) Quantification of TUNELpositive and EdU-positive Hep3B cells after knock-in of wild-type P53 with/without administration of UDCA. (ns: not significant, $p>0.05 ; *, p<0.05 ; * *, p<0.01 ; * * *, p<0.001$ ).
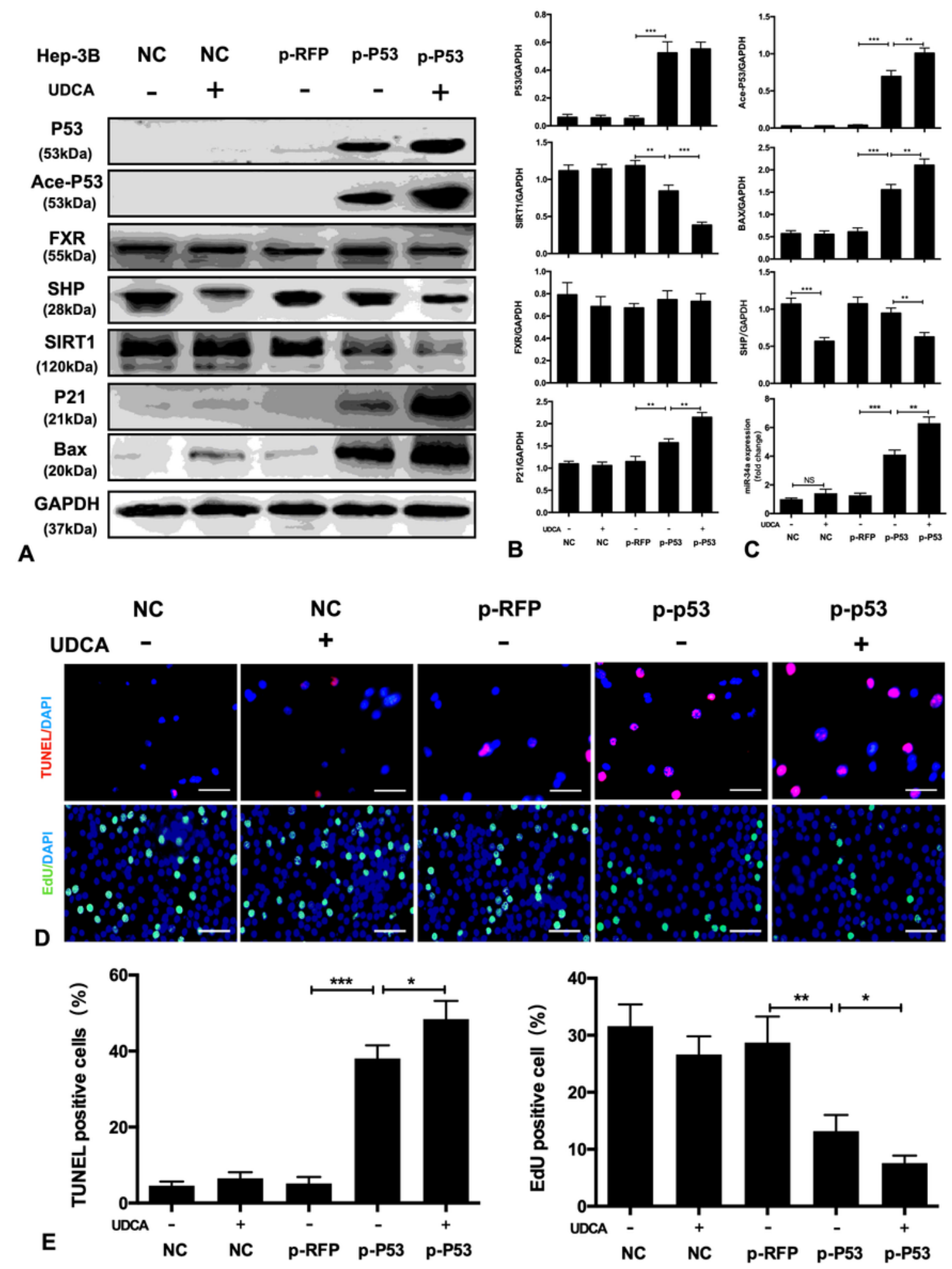

Figure 8 
Knock-in of wild-type P53 restored the P53/miR-34a/SIRT1 positive feedback in P53-deficient Hep-3B cell lines. (A) Protein expression of P53/miR-34a/SIRT1 positive feedback loop genes and FXR/SHP signaling after knock-in of wild-type P53 with/without administration of UDCA in P53-deficient Hep3B cells. Cells were pretreated with/without $200 \mu \mathrm{M}$ UDCA for $2 \mathrm{~h}$ before the experiment. (B) Quantification of P53, AceP53, SIRT1, P21, BAX, FXR, and SHP protein expression by WB (C) Quantification of miR-34a expression in P53-deficient Hep3B cells after knock-in of wild-type P53 with/without administration of UDCA. (D) TUNEL and EdU staining of P53-deficient Hep3B cells after knock-in of wild-type P53 with/without administration of UDCA (magnification: $\times 400$, Scale bars represent $50 \mu \mathrm{m}$ ). (E) Quantification of TUNELpositive and EdU-positive Hep3B cells after knock-in of wild-type P53 with/without administration of UDCA. (ns: not significant, $p>0.05 ; *, p<0.05 ; * \star, p<0.01 ; * \star \star, p<0.001$ ).

\section{Supplementary Files}

This is a list of supplementary files associated with this preprint. Click to download.

- figureS1.jpg

- figureS1.jpg

- figs2.jp2

- figs2.jp2

- FigS3.jp2

- FigS3.jp2

- FigS4.jp2

- FigS4.jp2

- SupplementaryCB.docx

- SupplementaryCB.docx

- GraphicalAbstract.Png

- GraphicalAbstract.Png 